\title{
Strange Baryon Production in Z Hadronic Decays
}

DELPHI Collaboration

\begin{abstract}
A study of the production of strange octet and decuplet baryons in hadronic decays of the $\mathrm{Z}$ recorded by the DELPHI detector at LEP is presented. This includes the first measurement of the $\Sigma^{ \pm}$average multiplicity. The total and differential cross sections, the event topology and the baryon-antibaryon correlations are compared with current hadronization models.
\end{abstract}

(To be submitted to Zeit. f. Phys. C) 
P.Abreu ${ }^{21}$, W.Adam ${ }^{50}$, T.Adye ${ }^{37}$, E.Agasi ${ }^{31}$, I.Ajinenko ${ }^{42}$, R.Aleksan ${ }^{39}$, G.D.Alekseev ${ }^{16}$, P.P.Allport ${ }^{22}$, S.Almehed $^{24}$, S.J.Alvsvaag ${ }^{4}$, U.Amaldi ${ }^{9}$, S.Amato ${ }^{47}$, A.Andreazza ${ }^{28}$, M.L.Andrieux ${ }^{14}$, P.Antilogus ${ }^{25}$, V.Anykeyev $^{42}$, W-D.Apel ${ }^{17}$, Y.Arnoud ${ }^{39}, \quad$ B.Asman ${ }^{44}$, J-E.Augustin ${ }^{19}$, A.Augustinus ${ }^{31}, \quad$ P.Baillon ${ }^{9}$, P.Bambade $^{19}$, R.Barate ${ }^{14}$, D.Y.Bardin ${ }^{16}$, G.J.Barker ${ }^{35}$, A.Baroncelli ${ }^{40}$, O.Barring ${ }^{24}$, J.A.Barrio ${ }^{26}$, W.Bartl ${ }^{50}$, M.J.Bates ${ }^{37}$, M.Battaglia ${ }^{15}$, M.Baubillier ${ }^{23}$, J.Baudot ${ }^{39}, \quad$ K-H.Becks ${ }^{52}$, M.Begalli ${ }^{6}$, P.Beilliere ${ }^{8}$, Yu.Belokopytov $^{9}$, A.C.Benvenuti ${ }^{5}$, M.Berggren ${ }^{41}$, D.Bertrand ${ }^{2}$, F.Bianchi ${ }^{45}$, M.Bigi ${ }^{45}$, M.S.Bilenky $^{16}$, $^{4}$, P.Billoir ${ }^{23}$, D.Bloch ${ }^{10}$, M.Blume ${ }^{52}$, S.Blyth ${ }^{35}$, V.Bocci ${ }^{38}$, T.Bolognese ${ }^{39}$, M.Bonesini ${ }^{28}$, W.Bonivento ${ }^{28}$, P.S.L.Booth ${ }^{22}$, G.Borisov ${ }^{42}$, C.Bosio ${ }^{40}$, S.Bosworth ${ }^{35}$, O.Botner ${ }^{48}$, E.Boudinov ${ }^{42}$, B.Bouquet ${ }^{19}$, C.Bourdarios $^{9}$, T.J.V.Bowcock ${ }^{22}$, M.Bozzo ${ }^{13}$, P.Branchini ${ }^{40}$, K.D.Brand ${ }^{36}$, R.A.Brenner ${ }^{15}$, C.Bricman ${ }^{2}$, L.Brillault ${ }^{23}$, R.C.A.Brown ${ }^{9}$, P.Bruckman ${ }^{18}$, J-M.Brunet ${ }^{8}$, L.Bugge ${ }^{33}$, T.Buran $^{33}$, A.Buys ${ }^{9}$, M.Caccia $^{28}$, M.Calvi $^{28}$, A.J.Camacho Rozas ${ }^{41}$, T.Camporesi ${ }^{9}$, V.Canale ${ }^{38}$, M.Canepa ${ }^{13}$, K.Cankocak ${ }^{44}$, F.Cao ${ }^{2}$, F.Carena ${ }^{9}$, P.Carrilho $^{47}$, L.Carroll ${ }^{22}$, C.Caso ${ }^{13}$, V.Cassio ${ }^{45}$, M.V.Castillo Gimenez ${ }^{49}$, A.Cattai ${ }^{9}$, F.R.Cavallo ${ }^{5}$, L.Cerrito $^{38}$, V.Chabaud $^{9}$, Ph.Charpentier ${ }^{9}$, L.Chaussard ${ }^{25}$, J.Chauveau ${ }^{23}$, P.Checchia ${ }^{36}$, G.A.Chelkov ${ }^{16}$, P.Chliapnikov ${ }^{42}$, P.Chochula $^{7}$, V.Chorowicz ${ }^{9}$, V.Cindro ${ }^{43}$, P.Collins ${ }^{9}$, J.L.Contreras ${ }^{19}$, R.Contri ${ }^{13}$, E.Cortina ${ }^{49}$, G.Cosme $^{19}$, F.Cossutti ${ }^{46}$, H.B.Crawley ${ }^{1}$, D.Crennell ${ }^{37}$, G.Crosetti ${ }^{13}$, J.Cuevas Maestro ${ }^{34}$, S.Czellar ${ }^{15}$, E.Dahl-Jensen ${ }^{29}$, J.Dahm $^{52}$, B.Dalmagne ${ }^{19}$, M.Dam ${ }^{33}$, G.Damgaard ${ }^{29}$, A.Daum ${ }^{17}$, P.D.Dauncey ${ }^{37}$, M.Davenport ${ }^{9}$, W.Da Silva ${ }^{23}$, C.Defoix $^{8}$, G.Della Ricca ${ }^{46}$, P.Delpierre ${ }^{27}$, N.Demaria ${ }^{35}$, A.De Angelis ${ }^{9}$, H.De Boeck ${ }^{2}$, W.De Boer ${ }^{17}$, S.De Brabandere ${ }^{2}$ C.De Clercq ${ }^{2}$, C.De La Vaissiere ${ }^{23}$, B.De Lotto ${ }^{46}$, A.De Min ${ }^{28}$, L.De Paula ${ }^{47}$, C.De Saint- $J_{e a n}{ }^{39}$, H.Dijkstra ${ }^{9}$, L.Di Ciaccio ${ }^{38}$, F.Djama $^{10}$, J.Dolbeau ${ }^{8}$, M.Donszelmann ${ }^{9}$, K.Doroba ${ }^{51}$, M.Dracos ${ }^{10}$, J.Drees ${ }^{52}$, K.-A.Drees ${ }^{52}$, M.Dris ${ }^{32}$, Y.Dufour ${ }^{8}$, F.Dupont ${ }^{14}$, D.Edsall ${ }^{1}$, R.Ehret ${ }^{17}$, G.Eigen ${ }^{4}$, T.Ekelof $^{48}$, G.Ekspong ${ }^{44}$, M.Elsing ${ }^{52}$, J-P.Engel ${ }^{10}$, N.Ershaidat ${ }^{23}$, B.Erzen ${ }^{43}$, M.Espirito Santo ${ }^{21}$, E.Falk ${ }^{24}$, D.Fassouliotis ${ }^{32}$, M.Feindt ${ }^{9}$, A.Ferrer ${ }^{49}$, T.A.Filippas ${ }^{32}$, A.Firestone ${ }^{1}$, H.Foeth ${ }^{9}$, E.Fokitis ${ }^{32}$, F.Fontanelli3 ${ }^{13}$, F.Formenti $^{9}$, B.Franek ${ }^{37}$, P.Frenkiel ${ }^{8}$, D.C.Fries ${ }^{17}$, A.G.Frodesen ${ }^{4}$, R.Fruhwirth ${ }^{50}$, F.Fulda-Quenzer ${ }^{19}$, H.Furstenau ${ }^{9}$, J.Fuster ${ }^{49}$, D.Gamba ${ }^{45}$, M.Gandelman ${ }^{6}$, C.Garcia ${ }^{49}$, J.Garcia ${ }^{41}$, C.Gaspar $^{9}$, U.Gasparini $^{36}$, Ph.Gavillet $^{9}$, E.N.Gazis ${ }^{32}$, D.Gele ${ }^{10}$, J-P.Gerber ${ }^{10}$, D.Gillespie ${ }^{9}$, R.Gokieli ${ }^{51}$, B.Golob ${ }^{43}$, G.Gopal ${ }^{37}$, L.Gorn ${ }^{1}$, M.Gorski ${ }^{51}$, Y.Gouz ${ }^{42}$, V.Gracco ${ }^{13}$, F.Grard ${ }^{2}$, E.Graziani ${ }^{40}$, G.Grosdidier ${ }^{19}$, P.Gunnarsson ${ }^{44}$, M.Gunther $^{48}$, J.Guy $^{37}$, U.Haedinger ${ }^{17}$, F.Hahn ${ }^{52}$, M.Hahn ${ }^{17}$, S.Hahn ${ }^{52}$, S.Haider ${ }^{31}$, Z.Hajduk ${ }^{18}$, A.Hallgren ${ }^{48}$, K.Hamacher $^{52}$, W.Hao $^{31}$, F.J.Harris ${ }^{35}$, V.Hedberg ${ }^{24}$, R.Henriques ${ }^{21}$, J.J.Hernandez ${ }^{49}$, P.Herquet ${ }^{2}$, H.Herr ${ }^{9}$, T.L.Hessing ${ }^{9}$, E.Higon $^{49}$, H.J.Hilke ${ }^{9}$, T.S.Hill ${ }^{1}$, S-O.Holmgren ${ }^{44}$, P.J.Holt ${ }^{35}$, D.Holthuizen ${ }^{31}$, M.Houlden ${ }^{22}$, J.Hrubec $^{50}$, K.Huet $^{2}$, K.Hultqvist ${ }^{44}$, P.Ioannou ${ }^{3}$, J.N.Jackson ${ }^{22}$, R.Jacobsson ${ }^{44}$, P.Jalocha ${ }^{18}$, R.Janik ${ }^{7}$, G.Jarlskog ${ }^{24}$, P.Jarry $^{39}$, B.Jean-Marie ${ }^{19}$, E.K.Johansson ${ }^{44}$, L.Jonsson ${ }^{24}$, P.Jonsson ${ }^{24}$, C.Joram ${ }^{9}$, P.Juillot ${ }^{10}$, M.Kaiser $^{17}$, G.Kalmus $^{37}$, F.Kapusta ${ }^{23}$, M.Karlsson ${ }^{44}$, E.Karvelas ${ }^{11}$, S.Katsanevas ${ }^{3}$, E.C.Katsoufis ${ }^{32}$, R.Keranen ${ }^{15}$, B.A.Khomenko ${ }^{16}$, N.N.Khovanski ${ }^{16}$, B.King ${ }^{22}$, N.J.Kjaer ${ }^{29}$, H.Klein ${ }^{9}$, A.Klovning ${ }^{4}$, P.Kluit ${ }^{31}$, J.H.Koehne $^{17}$, B.Koene ${ }^{31}$, P.Kokkinias ${ }^{11}$, M.Koratzinos ${ }^{9}$, K.Korcyl ${ }^{18}$, V.Kostioukhine ${ }^{42}$, C.Kourkoumelis ${ }^{3}$, O.Kouznetsov ${ }^{13}{ }$, P.-H.Kramer ${ }^{52}$, M.Krammer ${ }^{50}$, C.Kreuter ${ }^{17}$, J.Krolikowski ${ }^{51}$, I.Kronkvist ${ }^{24}$, Z.Krumstein ${ }^{16}$, W.Krupinski ${ }^{18}$, P.Kubinec $^{7}$, W.Kucewicz ${ }^{18}$, K.Kurvinen ${ }^{15}$, C.Lacasta $^{49}$, I.Laktineh ${ }^{25}$, S.Lamblot ${ }^{23}$, J.W.Lamsa ${ }^{1}$, L.Lanceri ${ }^{46}{ }^{4}$, D.W.Lane ${ }^{1}$, P.Langefeld ${ }^{52}$, V.Lapin ${ }^{42}$, I.Last ${ }^{22}$, J-P.Laugier ${ }^{39}$, R.Lauhakangas ${ }^{15}$, G.Leder $^{50}$, F.Ledroit $^{14}$, V.Lefebure $^{2}$, C.K.Legan ${ }^{1}$, R.Leitner ${ }^{30}$, Y.Lemoigne ${ }^{39}$, J.Lemonne ${ }^{2}$, G.Lenzen ${ }^{52}$, V.Lepeltier ${ }^{19}$, T.Lesiak ${ }^{36}$, D.Liko $^{50}$, R.Lindner ${ }^{52}$, A.Lipniacka ${ }^{19}$, I.Lippi ${ }^{36}$, B.Loerstad ${ }^{24}$, M.Lokajicek ${ }^{12}$, J.G.Loken ${ }^{35}$, J.M.Lopez ${ }^{41}$, A.Lopez-Fernandez ${ }^{9}$, M.A.Lopez Aguera ${ }^{41}$, D.Loukas ${ }^{11}$, P.Lutz ${ }^{39}$, L.Lyons ${ }^{35}$, J.MacNaughton ${ }^{50}$, G.Maehlum $^{17}$, A.Maio $^{21}$, V.Malychev ${ }^{16}$, F.Mandl ${ }^{50}$, J.Marco ${ }^{41}$, B.Marechal ${ }^{47}$, M.Margoni ${ }^{36}$, J-C.Marin ${ }^{9}$, C.Mariotti ${ }^{40}$, A.Markou ${ }^{11}$, T.Maron ${ }^{52}$, C.Martinez-Rivero ${ }^{41}$, F.Martinez-Vidal ${ }^{49}$, S.Marti i Garcia ${ }^{49}$, F.Matorras ${ }^{41}$, C.Matteuzzi $^{28}$, G.Matthiae ${ }^{38}$, M.Mazzucato ${ }^{36}$, M.Mc Cubbin ${ }^{9}$, R.Mc Kay ${ }^{1}$, R.Mc Nulty ${ }^{22}$, J.Medbo ${ }^{48}$, C.Meroni $^{28}$, W.T.Meyer ${ }^{1}$, M.Michelotto ${ }^{36}$, E.Migliore ${ }^{45}$, L.Mirabito ${ }^{25}$, W.A.Mitaroff ${ }^{50}$, U.Mjoernmark ${ }^{24}$, T.Moa $^{44}$, R.Moeller ${ }^{29}$, K.Moenig ${ }^{9}$, M.R.Monge ${ }^{13}$, P.Morettini ${ }^{13}$, H.Mueller ${ }^{17}$, L.M.Mundim ${ }^{6}$, W.J.Murray ${ }^{37}$, B.Muryn $^{18}$, G.Myatt ${ }^{35}$, F.Naraghi ${ }^{14}$, F.L.Navarria ${ }^{5}$, S.Navas ${ }^{49}$, P.Negri ${ }^{28}$, S.Nemecek ${ }^{12}$, W.Neumann ${ }^{52}$, N.Neumeister ${ }^{50}$, R.Nicolaidou ${ }^{3}$, B.S.Nielsen ${ }^{29}$, V.Nikolaenko ${ }^{10}$, P.Niss ${ }^{44}$, A.Nomerotski ${ }^{36}$, A.Normand ${ }^{35}$, W.Oberschulte-Beckmann ${ }^{17}$, V.Obraztsov ${ }^{42}$, A.G.Olshevski ${ }^{16}$, A.Onofre ${ }^{21}$, R.Orava ${ }^{15}$, K.Osterberg ${ }^{15}$, A.Ouraou $^{39}$, P.Paganini ${ }^{19}$, M.Paganoni ${ }^{28}$, P.Pages ${ }^{10}$, H.Palka ${ }^{18}$, Th.D.Papadopoulou ${ }^{32}$, L.Pape ${ }^{9}$, F.Parodi ${ }^{13}$,

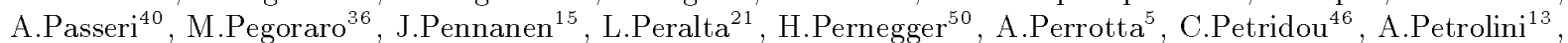
H.T.Phillips ${ }^{37}$, G.Piana ${ }^{13}$, F.Pierre ${ }^{39}$, M.Pimenta ${ }^{21}$, S.Plaszczynski ${ }^{19}$, O.Podobrin ${ }^{17}$, M.E.Pol ${ }^{6}$, G.Polok $^{18}{ }^{\text {, }}$ P.Poropat $^{46}$, V.Pozdniakov ${ }^{16}$, M.Prest ${ }^{46}$, P.Privitera ${ }^{38}$, A.Pullia ${ }^{28}$, D.Radojicic ${ }^{35}$, S.Ragazzi ${ }^{28}$, H.Rahmani $^{32}$, J.Rames $^{12}$, P.N.Ratoff ${ }^{20}$, A.L.Read ${ }^{33}$, M.Reale ${ }^{52}$, P.Rebechii ${ }^{19}$, N.G.Redaelli ${ }^{28}$, M.Regler ${ }^{50}$, D.Reid ${ }^{9}$, P.B.Renton $^{35}$, L.K.Resvanis ${ }^{3}$, F.Richard ${ }^{19}$, J.Richardson ${ }^{22}$, J.Ridky ${ }^{12}$, G.Rinaudo ${ }^{45}$, I.Ripp ${ }^{39}$, A.Romero ${ }^{45}$, I.Roncagliolo $^{13}$, P.Ronchese ${ }^{36}$, L.Roos ${ }^{14}$, E.I.Rosenberg ${ }^{1}$, E.Rosso ${ }^{9}$, P.Roudeau ${ }^{19}$, T.Rovelli ${ }^{5}$, W.Ruckstuhl ${ }^{31}$, V.Ruhlmann-Kleider ${ }^{39}$, A.Ruiz ${ }^{41}$, H.Saarikko ${ }^{15}$, Y.Sacquin ${ }^{39}$, A.Sadovsky ${ }^{16}$, G.Sajot ${ }^{14}$, J.Salt ${ }^{49}$, J.Sanchez ${ }^{26}$, M.Sannino ${ }^{13}$, H.Schneider ${ }^{17}$, M.A.E.Schyns ${ }^{52}$, G.Sciolla ${ }^{45}$, F.Scuri ${ }^{46}$, Y.Sedykh ${ }^{16}$, A.M.Segar ${ }^{35}$, A.Seitz ${ }^{17}$, R.Sekulin ${ }^{37}$, R.C.Shellard ${ }^{6}$, I.Siccama ${ }^{31}$, P.Siegrist ${ }^{39}$, S.Simonetti ${ }^{39}$, F.Simonetto ${ }^{36}$, A.N.Sisakian ${ }^{16}$, B.Sitar $^{7}$, T.B.Skaali ${ }^{33}$, G.Smadja ${ }^{25}$, N.Smirnov ${ }^{42}$, O.Smirnova ${ }^{16}$, G.R.Smith ${ }^{37}$, R.Sosnowski ${ }^{51}$, D.Souza-Santos ${ }^{6}$, T.Spassov ${ }^{21}$, E.Spiriti ${ }^{40}$, S.Squarcia ${ }^{13}$, H.Staeck ${ }^{52}$, C.Stanescu ${ }^{40}$, S.Stapnes ${ }^{33}$, I.Stavitski ${ }^{36}$, K.Stepaniak ${ }^{51}$, F.Stichelbaut $^{9}$, A.Stocchi ${ }^{19}$, J.Strauss ${ }^{50}$, R.Strub ${ }^{10}$, B.Stugu ${ }^{4}$, M.Szczekowski ${ }^{51}$, M.Szeptycka ${ }^{51}$, T.Tabarelli ${ }^{28}$, J.P.Tavernet ${ }^{23}$, O.Tchikilev ${ }^{42}$, A.Tilquin ${ }^{27}$, J.Timmermans ${ }^{31}$, L.G.Tkatchev ${ }^{16}$, T.Todorov ${ }^{10}$, D.Z.Toet $^{31}$, A.Tomaradze $^{2}$, B.Tome ${ }^{21}$, L.Tortora ${ }^{40}$, G.Transtromer ${ }^{24}$, D.Treille ${ }^{9}$, W.Trischuk ${ }^{9}$, G.Tristram ${ }^{8}$, A.Trombini ${ }^{19}$, 
C.Troncon ${ }^{28}$, A.Tsirou ${ }^{9}, \quad$ M-L.Turluer ${ }^{39}$, T.Tuuva ${ }^{15}$, I.A.Tyapkin ${ }^{16}, \quad$ M.Tyndel ${ }^{37}, \quad$ S.Tzamarias ${ }^{22}$, B.Ueberschaer $^{52}$, S.Ueberschaer ${ }^{52}$, O.Ullaland ${ }^{9}$, V.Uvarov ${ }^{42}$, G.Valenti ${ }^{5}$, E.Vallazza ${ }^{9}$, C.Vander Velde ${ }^{2}$. G.W.Van Apeldoorn ${ }^{31}$, P.Van Dam ${ }^{31}$, W.K.Van Doninck ${ }^{2}$, J.Van Eldik ${ }^{31}$, G.Vegni ${ }^{28}$, L.Ventura ${ }^{36}$, W.Venus $^{37}$, F.Verbeure $^{2}$, M.Verlato ${ }^{36}$, L.S.Vertogradov ${ }^{16}$, D.Vilanova ${ }^{39}$, P.Vincent ${ }^{25}$, L.Vitale $^{46}$, $_{\text {E.Vlasov }}{ }^{42}$, A.S.Vodopyanov ${ }^{16}$, M.Voutilainen ${ }^{15}$, V.Vrba $^{12}$, H.Wahlen ${ }^{52}$, C.Walck ${ }^{44}$, F.Waldner ${ }^{46}$, A.Wehr ${ }^{52}$, M.Weierstall $^{52}$, P.Weilhammer ${ }^{9}$, A.M.Wetherell ${ }^{9}$, D.Wicke ${ }^{52}$, J.H.Wickens ${ }^{2}$, M.Wielers ${ }^{17}$, G.R.Wilkinson ${ }^{35}$, W.S.C.Williams ${ }^{35}$, M.Winter ${ }^{10}$, M.Witek $^{9}$, G.Wormser ${ }^{19}$, K.Woschnagg ${ }^{48}$, K.Yip ${ }^{35}$, L.Yu ${ }^{35}$, O.Yushchenko ${ }^{42}$, F.Zach $^{25}$, C.Zacharatou ${ }^{24}$, A.Zalewska ${ }^{18}$, P.Zalewski $^{51}$, D.Zavrtanik ${ }^{43}$, E.Zevgolatakos ${ }^{11}$, V.Zhigunov $^{42}$, N.I.Zimin ${ }^{16}$, M.Zito ${ }^{39}$, D.Zontar ${ }^{43}$, R.Zuberi ${ }^{35}$, G.C.Zucchelli ${ }^{44}$, G.Zumerle ${ }^{36}$

\footnotetext{
${ }^{1}$ Ames Laboratory and Department of Physics, Iowa State University, Ames IA 50011, USA

${ }^{2}$ Physics Department, Univ. Instelling Antwerpen, Universiteitsplein 1, B-2610 Wilrijk, Belgium and IIHE, ULB-VUB, Pleinlaan 2, B-1050 Brussels, Belgium and Faculté des Sciences, Univ. de l'Etat Mons, Av. Maistriau 19, B-7000 Mons, Belgium ${ }^{3}$ Physics Laboratory, University of Athens, Solonos Str. 104, GR-10680 Athens, Greece ${ }^{4}$ Department of Physics, University of Bergen, Allégaten 55, N-5007 Bergen, Norway ${ }^{5}$ Dipartimento di Fisica, Università di Bologna and INFN, Via Irnerio 46, I-40126 Bologna, Italy ${ }^{6}$ Centro Brasileiro de Pesquisas Físicas, rua Xavier Sigaud 150, RJ-22290 Rio de Janeiro, Brazil and Depto. de Física, Pont. Univ. Católica, C.P. 38071 RJ-22453 Rio de Janeiro, Brazil and Inst. de Física, Univ. Estadual do Rio de Janeiro, rua São Francisco Xavier 524, Rio de Janeiro, Brazil ${ }^{7}$ Comenius University, Faculty of Mathematics and Physics, Mlynska Dolina, SK-84215 Bratislava, Slovakia ${ }^{8}$ Collège de France, Lab. de Physique Corpusculaire, IN2P3-CNRS, F-75231 Paris Cedex 05, France

${ }^{9}$ CERN, CH-1211 Geneva 23, Switzerland

${ }^{10}$ Centre de Recherche Nucléaire, IN2P3 - CNRS/ULP - BP20, F-67037 Strasbourg Cedex, France

${ }^{11}$ Institute of Nuclear Physics, N.C.S.R. Demokritos, P.O. Box 60228, GR-15310 Athens, Greece

${ }^{12}$ FZU, Inst. of Physics of the C.A.S. High Energy Physics Division, Na Slovance 2, 180 40, Praha 8, Czech Republic

${ }^{13}$ Dipartimento di Fisica, Università di Genova and INFN, Via Dodecaneso 33, I-16146 Genova, Italy

${ }^{14}$ Institut des Sciences Nucléaires, IN2P3-CNRS, Université de Grenoble 1, F-38026 Grenoble Cedex, France

${ }^{15}$ Research Institute for High Energy Physics, SEFT, P.O. Box 9, FIN-00014 Helsinki, Finland

${ }^{16}$ Joint Institute for Nuclear Research, Dubna, Head Post Office, P.O. Box 79, 101000 Moscow, Russian Federation

${ }^{17}$ Institut für Experimentelle Kernphysik, Universität Karlsruhe, Postfach 6980, D-76128 Karlsruhe, Germany

${ }^{18}$ High Energy Physics Laboratory, Institute of Nuclear Physics, Ul. Kawiory 26a, PL-30055 Krakow 30, Poland

${ }^{19}$ Université de Paris-Sud, Lab. de l'Accélérateur Linéaire, IN2P3-CNRS, Bat 200, F-91405 Orsay Cedex, France

${ }^{20}$ School of Physics and Materials, University of Lancaster, Lancaster LA1 4YB, UK

${ }^{21}$ LIP, IST, FCUL - Av. Elias Garcia, 14-1 ${ }^{\circ}$, P-1000 Lisboa Codex, Portugal

${ }^{22}$ Department of Physics, University of Liverpool, P.O. Box 147, Liverpool L69 3BX, UK

${ }^{23}$ LPNHE, IN2P3-CNRS, Universités Paris VI et VII, Tour 33 (RdC), 4 place Jussieu, F-75252 Paris Cedex 05, France

${ }^{24}$ Department of Physics, University of Lund, Sölvegatan 14, S-22363 Lund, Sweden

${ }^{25}$ Université Claude Bernard de Lyon, IPNL, IN2P3-CNRS, F-69622 Villeurbanne Cedex, France

${ }^{26}$ Universidad Complutense, Avda. Complutense s/n, E-28040 Madrid, Spain

${ }^{27}$ Univ. d'Aix - Marseille II - CPP, IN2P3-CNRS, F-13288 Marseille Cedex 09, France

${ }^{28}$ Dipartimento di Fisica, Università di Milano and INFN, Via Celoria 16, I-20133 Milan, Italy

${ }^{29}$ Niels Bohr Institute, Blegdamsvej 17, DK-2100 Copenhagen 0, Denmark

${ }^{30}$ NC, Nuclear Centre of MFF, Charles University, Areal MFF, V Holesovickach 2, 180 00, Praha 8, Czech Republic

${ }^{31}$ NIKHEF-H, Postbus 41882, NL-1009 DB Amsterdam, The Netherlands

${ }^{32}$ National Technical University, Physics Department, Zografou Campus, GR-15773 Athens, Greece

${ }^{33}$ Physics Department, University of Oslo, Blindern, N-1000 Oslo 3, Norway

${ }^{34}$ Dpto. Fisica, Univ. Oviedo, C/P. Pérez Casas, S/N-33006 Oviedo, Spain

${ }^{35}$ Department of Physics, University of Oxford, Keble Road, Oxford OX1 3RH, UK

${ }^{36}$ Dipartimento di Fisica, Università di Padova and INFN, Via Marzolo 8, I-35131 Padua, Italy

${ }^{37}$ Rutherford Appleton Laboratory, Chilton, Didcot OX11 OQX, UK

${ }^{38}$ Dipartimento di Fisica, Università di Roma II and INFN, Tor Vergata, I-00173 Rome, Italy

${ }^{39}$ Centre d'Etude de Saclay, DSM/DAPNIA, F-91191 Gif-sur-Yvette Cedex, France

${ }^{40}$ Istituto Superiore di Sanità, Ist. Naz. di Fisica Nucl. (INFN), Viale Regina Elena 299, I-00161 Rome, Italy

${ }^{41}$ C.E.A.F.M., C.S.I.C. - Univ. Cantabria, Avda. los Castros, S/N-39006 Santander, Spain, (CICYT-AEN93-0832)

${ }^{42}$ Inst. for High Energy Physics, Serpukov P.O. Box 35, Protvino, (Moscow Region), Russian Federation

${ }^{43} \mathrm{~J}$. Stefan Institute and Department of Physics, University of Ljubljana, Jamova 39, SI-61000 Ljubljana, Slovenia

${ }^{44}$ Fysikum, Stockholm University, Box 6730, S-113 85 Stockholm, Sweden

${ }^{45}$ Dipartimento di Fisica Sperimentale, Università di Torino and INFN, Via P. Giuria 1, I-10125 Turin, Italy

${ }^{46}$ Dipartimento di Fisica, Università di Trieste and INFN, Via A. Valerio 2, I-34127 Trieste, Italy and Istituto di Fisica, Università di Udine, I-33100 Udine, Italy

${ }^{47}$ Univ. Federal do Rio de Janeiro, C.P. 68528 Cidade Univ., Tlha do Fundão BR-21945-970 Rio de Janeiro, Brazil

${ }^{48}$ Department of Radiation Sciences, University of Uppsala, P.O. Box 535, S-751 21 Uppsala, Sweden

${ }^{49}$ IFIC, Valencia-CSIC, and D.F.A.M.N., U. de Valencia, Avda. Dr. Moliner 50, E-46100 Burjassot (Valencia), Spain

${ }^{50}$ Institut für Hochenergiephysik, Österr. Akad. d. Wissensch., Nikolsdorfergasse 18, A-1050 Vienna, Austria

${ }^{51}$ Inst. Nuclear Studies and University of Warsaw, Ul. Hoza 69, PL-00681 Warsaw, Poland

${ }^{52}$ Fachbereich Physik, University of Wuppertal, Postfach 100 127, D-42097 Wuppertal 1, Germany
} 


\section{Introduction}

The phenomenological interpretation of the production of the $S=-1$ and $S=-2$ baryons in $e^{+} e^{-}$annihilation at the $\mathrm{Z}$ is based on the formation of $\mathrm{S}=-1,-2$ diquarks [1] during hadronization. These are objects of spin 0 or 1 and contain one or more strange quarks. However there are several free parameters in the hadronization models and it might be expected that by judicious tuning, the predictions can converge on an empirically adequate description of the process of partons fragmenting into hadrons. To check the adequacy of such models it is necessary that the baryon production be studied in the context of the overall relevant hadron production.

In this paper the production dynamics of the $\Xi^{-}, \Xi(1530)^{0}, \Sigma(1385)^{ \pm}$and $\Sigma^{ \pm}$baryons are studied and compared to the $\Lambda$ and $\mathrm{K}^{0}$ production properties. The measured total cross sections are compared with the JETSET 7.3 [2] and HERWIG 5.7 [3] predictions. The jet structure of the hadronic events containing the particles and the correlations in baryon production are also studied.

The present work extends the results of our previous [4] DELPHI publication on the $\Xi^{-}$production and together with $[5,6]$ covers a broad range of topics concerning strange meson and baryon production in $\mathrm{Z}$ hadronic decays.

\section{Experimental Procedure and Event Selection}

A description of the DELPHI detector can be found in Ref. [7]. The present analysis is based on the information provided by the central tracking detectors: the Micro-Vertex Detector (VD), the Inner Detector (ID), the Time Projection Chamber (TPC) and the Outer Detector (OD); and by the Hadronic Calorimeter (HCAL).

The central DELPHI tracking detectors cover the region between $25^{\circ}$ and $155^{\circ}$ in polar angle $\theta$. These give an average momentum resolution of charged particles in hadronic $\mathrm{Z}$ decays in the range $\Delta p / p \sim 0.001 \times p$ to $\Delta p / p \sim 0.01 \times p(p$ in $\mathrm{GeV} / c)$, depending on which detectors are included in the track fit.

Charged particles were included in this analysis if they had:

- track length measured in the TPC > $30 \mathrm{~cm}$;

- momentum between $0.1 \mathrm{GeV} / c$ and $35 \mathrm{GeV} / c$;

- relative error on the momentum measurement less than $100 \%$;

- polar angle of the track, $\theta$, between 25 and 155 degrees.

Hadronic $\mathrm{Z}$ decays were selected by requiring :

- at least seven charged tracks $\left(N_{c h} \geq 7\right)$;

- energy sum of the charged tracks (assumed to be pions) in each hemisphere greater than $3 \mathrm{GeV}\left(\sum E_{c h}^{ \pm}>3 \mathrm{GeV}\right)$;

- total energy sum of the charged tracks greater than $15 \mathrm{GeV}\left(\sum E_{c h}^{ \pm}>15 \mathrm{GeV}\right)$.

A total of 950000 events from the 1991 and 1992 runs (for the $\Xi(1530)^{0}$ analysis a sample of 1.75 million events from the 1991,1992 and 1993 runs) satisfied these cuts. Events due to the beam gas scattering and to $\gamma \gamma$ interactions have been estimated to be less than $0.1 \%$ of the sample, while background from $\tau^{+} \tau^{-}$pairs was calculated to be less than $0.2 \%$.

In order to determine the position of the interaction point a multi-event vertex was formed (in the following denoted the 'primary vertex'). Tracks with transverse momentum with respect to the beam axis greater than $1 \mathrm{GeV} / c$ and 2 or more VD hits were 
selected from consecutive hadronic $Z$ decays (typically 3000 events, 2.5 tracks per event in average). These tracks were used to define a vertex in the plane perpendicular to the beam direction (the $\mathrm{R} \phi$ plane). The real interaction point for the 1991(1992) events has been estimated to lie within $10(10) \mu \mathrm{m}$ in the vertical direction and $150(100) \mu \mathrm{m}$ in the horizontal direction of this vertex at the one sigma level.

The influence of the detector resolution on this analysis was studied with the simulation program DELSIM [8] using as event generator JETSET 7.3 Parton Shower [2] with parameters tuned as in [9].

\section{$3 \quad \Lambda$ Reconstruction}

The following baryons ${ }^{1}$ were identified through their decays:

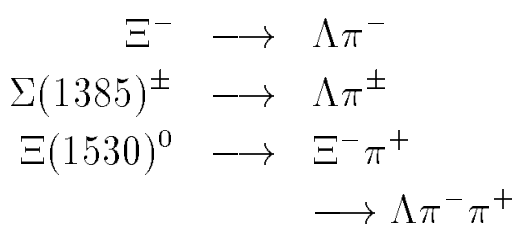

A first enrichment of the data sample was performed by requiring the identification of at least one $\Lambda$ candidate per event.

For any pair of oppositely charged tracks both intersections in the $\mathrm{R} \phi$ plane were taken as vertex candidates. The intersection for which the tracks had the minimum separation along the axis parallel to the beam direction (in the following the $z$ axis) was then selected. Pairs of tracks which did not intersect were also retained if they approached each other within $0.3 \mathrm{~cm}$. For all these tracks, a vertex fit was performed as described in [10]. The following cuts were applied to keep the maximum number of $\Lambda$ 's produced from hyperon decays whilst simultaneously reducing the combinatorial background:

- separation of the tracks along the $z$ axis at the secondary vertex not larger than 0.75 $\mathrm{cm}$;

- distance between the primary and the secondary vertex in the $\mathrm{R} \phi$ plane greater than $3 \mathrm{~cm}$;

- minimum impact parameter with respect to the primary vertex (distance of closest approach to the primary vertex) on the $\mathrm{R} \phi$ plane of the two particles greater than $0.03 \mathrm{~cm}$, to exclude particles from the primary vertex;

- angle $\theta$ less than $100 \mathrm{mrad}$ (where $\theta$ is the angle between the $\Lambda$ candidate momentum direction and the vector pointing from the primary to the secondary vertex, projected onto the $R \phi$ plane);

- transverse momentum of the two particles with respect to the $\Lambda$ direction greater than $0.02 \mathrm{GeV} / c$ (to remove $\gamma$ conversions).

- $\Lambda$ candidates required to have momentum greater than $0.5 \mathrm{GeV} / c$.

The invariant mass of the selected candidates was calculated by assigning the proton mass to the particle with the higher momentum. The mass spectrum of the $\Lambda$ candidates is shown in Figure 1.

A fit was performed with the Breit-Wigner distribution for the signal and a polynomial distribution for the background. From this, $54625 \pm 437 \pm 601$ candidates with mass

\footnotetext{
${ }^{1}$ In the following when a baryon (or baryon decay) is mentioned the antibaryon (or antibaryon decay) is also meant unless explicitly stated.
} 
$1115.20 \pm 0.02 \pm 0.20 \mathrm{MeV} / c^{2}$ and width $5.5 \pm 0.7 \pm 0.6 \mathrm{MeV} / c^{2}$ are found. The systematic errors have been calculated by replacing the Breit-Wigner formula by two Gaussians with common mean, using either a first or a second order polynomial to parameterize the background and varying the choice of mass windows for the fit.

The $\Lambda$ production yield was then calculated by repeating the analysis chain described in $[4,5]$. The mean production multiplicity is found to be $0.357 \pm 0.003 \pm 0.017 \Lambda$ 's per hadronic $Z$ decay, reproducing our previous published results [5] but using a slightly more efficient reconstruction algorithm. Agreement was found also for the differential momentum distribution.

\section{$4 \quad \Xi^{-}$Reconstruction}

Any $\Lambda$ candidate with invariant mass between $1.11 \mathrm{GeV} / c^{2}$ and $1.12 \mathrm{GeV} / c^{2}$ was combined with any charged track, taken as a pion, in order to reconstruct a $\Xi^{-}$candidate (in the following these $\Lambda$ candidates are referred to as $V_{0}$ 's and the nominal $\Lambda$ mass 1115.68 $\mathrm{MeV} / \mathrm{c}^{2}[11]$ is assigned to them, independent of their invariant mass). A vertex was formed in the $\mathrm{R} \phi$ plane if the flight path of the $V_{0}$ intersected the charged track. A simulation study has shown that non intersecting topologies do not contribute significantly to the reconstructed signal.

A combination of a $V_{0}$ and a charged particle was required to fulfill the following requirements in order to be accepted as a $\Xi^{-}$candidate:

- separation along the $z$ direction of the $V_{0}$ 's flight path and the charged track at the $\mathrm{R} \phi$ intersection point less than $0.75 \mathrm{~cm}$ (if both the intersections were valid the one with the smaller $z$ separation was selected);

- intersection point between the primary vertex and the $V_{0}$ decay vertex;

- radial distance on the $\mathrm{R} \phi$ plane between the primary vertex and the intersection point greater than $2 \mathrm{~cm}$;

- impact parameter of the charged particle with respect to the primary vertex on the $\mathrm{R} \phi$ plane greater than $0.2 \mathrm{~cm}$;

- impact parameter of the reconstructed $\Xi^{-}$candidate with respect to the primary vertex on the $\mathrm{R} \phi$ plane less than $1 \mathrm{~cm}$.

The mass spectrum of the selected $\Xi^{-}$and $\Xi^{-}$candidates is shown in Figure 2a, where the points represent the right sign $\left(\Lambda \pi^{-}, \bar{\Lambda} \pi^{+}\right)$, while the shaded histogram corresponds to the unphysical $\left(\Lambda \pi^{+}, \bar{\Lambda} \pi^{-}\right)$wrong sign combinations. The latter is expected to be a good estimation of the background under the reconstructed $\Xi^{-}$'s.

A fit using a Breit-Wigner distribution for the signal and an empirical parameterization for the background yields $1375 \pm 50 \pm 69 \Xi^{-}$'s with a mean mass value of $1320.6 \pm 0.05 \pm 0.5 \mathrm{MeV} / c^{2}$ (in good agreement with the world average of $1321.32 \pm 0.13$ $\mathrm{MeV} / \mathrm{c}^{2}[11]$ ) and a width of $5.5 \pm 0.8 \pm 0.6 \mathrm{MeV} / \mathrm{c}^{2}$. The systematic errors have been calculated by replacing the Breit-Wigner formula by two Gaussians with common mean using different functions to parametrize the background and varying the choice of the mass window for the fit.

The same procedure has been performed dividing the data sample into $6 x_{p}$ regions $\left(x_{p}\right.$ is the $\Xi^{-}$momentum normalized to the beam energy). The $x_{p}$ dependent reconstruction efficiencies have been calculated by treating simulated $\mathrm{Z}$ decays as real data.

A search was made for evidence of correlated $\Xi^{-}$and $\bar{\Lambda}$ production. In Figure $2 b$ the $\bar{p} \pi^{+}\left(p \pi^{-}\right)$invariant mass distribution is shown on top of the $p \pi^{-}\left(\bar{p} \pi^{+}\right)$spectrum for 
those events containing a $\Xi^{-}\left(\bar{\Xi}^{+}\right)$candidate with mass between 1.31 and $1.33 \mathrm{GeV} / \mathrm{c}^{2}$. The charged particle pairs have been selected with the criteria described in Section 3. A clear peak of $87 \pm 10 \pm 8$ baryon-antibaryon pairs exceeds a peak of $23 \pm 7 \pm 4$ baryon-baryon pairs around the nominal $\Lambda$ mass.

\section{$5 \quad \Xi(1530)^{0}$ Reconstruction}

The $\Xi(1530)^{0}$ was identified by its decay to $\Xi^{-} \pi^{+}$. The $\Xi^{-}$candidates were selected with criteria similar to those of Section 4 requiring an invariant mass between 1.31 and $1.33 \mathrm{GeV} / \mathrm{c}^{2}$ and using a sample of 1.75 million events (corresponding to the 1991, 1992 and 1993 running periods). These candidates were then combined with all charged tracks taken as having the pion mass and were considered as a candidate $\Xi(1530)^{0}$ decay if the pion track satisfied the following criteria:

- momentum between 0.1 and $2 \mathrm{GeV} / c$;

- impact parameters on the $R \phi$ and $R z$ planes less than 3 standard deviations from zero;

- $\cos (\theta)$ greater than 0.8 , where $\theta$ is the angle to the $\Xi^{-}$;

- origin at the interaction point (see below).

The origin of the tracks was determined as follows. Once the $\Xi^{-}$has been reconstructed, a vertex was estimated using all tracks of the event except those from the $\Xi^{-}$decay. Tracks contributing too much to the $\chi^{2}$ of the vertex were excluded until a $\chi^{2}$ probability greater than $10^{-3}$ was reached. Tracks included in the final fit were considered as originating from the interaction point.

The invariant mass spectra of the "right" $\left(\Xi^{-} \pi^{+}, \bar{\Xi}^{+} \pi^{-}\right)$and the "wrong" sign combination $\left(\Xi^{-} \pi^{-}, \bar{\Xi}^{+} \pi^{+}\right)$are shown in Figure 3a. There is an apparent disagreement in the background levels of the above mass distribution which can be explained, according to the simulation, by flavor correlations during the hadronization process. This is due mainly to the correlated production of a $\Xi^{-}(s s d)$ with $\mathrm{K}^{+}(\bar{s} u)$, which contributes only to right sign combinations when the kaon is mistaken for the pion. Also $\Xi^{-}$correlations with $\mathrm{K}^{*+}$ or $\mathrm{K}^{* 0}$ preferentially contribute to the right sign combinations (by a factor $5 / 2$ ) whilst baryonic number and strangeness local conservation contributes to this asymmetry by a much smaller amount. Simulation studies have shown that the observed asymmetry in the data it is consistent with the JETSET predictions as it is shown in Figure $3 \mathrm{~b}$.

The right sign combinations mass spectrum was fitted by using a Gaussian distribution for the signal and the following background parametrization:

$$
F(x)=(x-1.461)^{a_{5}} \times \exp \left(a_{0}+a_{1} x+a_{2} x^{2}+a_{3} x^{3}+a_{4} x^{4}\right)
$$

The fit resulted in $312 \pm 32 \Xi(1530)^{0}$ 's with a mean mass of $1533.4 \pm 0.8 \mathrm{MeV} / \mathrm{c}^{2}$ and a RMS width of $7.6 \pm 0.8 \mathrm{MeV} / c^{2}$ which is compatible with the simulation expectation of $1531.6 \pm 0.6 \mathrm{MeV} / c^{2}$ and $8.2 \pm 0.5 \mathrm{MeV} / c^{2}$ for the mean mass and RMS width respectively. The overall efficiency for the $\Xi(1530)^{0}$ reconstruction was calculated by treating the simulated events in the same way as the real data and it was found to be $4.3 \pm 0.3 \%$. 


\section{$6 \quad \Sigma(1385)^{ \pm}$Reconstruction}

$\Sigma(1385)^{ \pm}$is identified by its decay into $\Lambda \pi^{ \pm}$. All the $V_{0}$ 's selected with the criteria of Section 3 were combined with all charged particles taken as pions. A combination was considered to be a $\Sigma(1385)^{ \pm}$candidate if it satisfied the following requirements :

- $V_{0}$ impact parameter with respect to the primary vertex on the $\mathrm{R} \phi$ plane less than $0.15 \mathrm{~cm}$;

- impact parameter of the charged particle with respect to the primary vertex on the $\mathrm{R} \phi$ plane less than $0.2 \mathrm{~cm}$;

- separation along the $z$ axis between the $V_{0}$ and the charged particle at their points of closest approach to the primary vertex less than $1.0 \mathrm{~cm}$;

- momentum of the $\Lambda$ greater than $1.5 \mathrm{GeV} / \mathrm{c}$

- opening angle between the two particles less than $60^{\circ}$.

The resulting mass spectrum is shown in Figure 4 where there is an excess of events concentrated around the $\Sigma(1385)^{ \pm}$and $\Xi^{-}$nominal masses. The total mass spectrum was fitted by a Breit-Wigner and a Gaussian distribution function to represent the $\Sigma(1385)^{ \pm}$ and $\Xi^{-}$mass peaks respectively whilst empirical parametrizations were used to describe the background shape. A total of $2683 \pm 196 \pm 208 \Sigma(1385)^{ \pm}$'s are seen, distributed around a mean mass of $1381.0 \pm 2.0 \pm 2.0 \mathrm{MeV} / c^{2}$ (in comparison with the world average mass values of $1382.8 \pm 0.4 \mathrm{MeV} / c^{2}$ and $1387.2 \pm 0.5 \mathrm{MeV} / c^{2}$ for the $\Sigma(1385)^{+}$and the $\Sigma(1385)^{-}$ baryons respectively [11]) with a width of $32.3 \pm 2.6 \pm 1.9 \mathrm{MeV} / c^{2}$, to be compared to the world averages of $35.8 \pm 0.8 \mathrm{MeV} / c^{2}$ for the $\Sigma(1385)^{+}$and $39.4 \pm 2.1 \mathrm{MeV} / c^{2}$ for the $\Sigma(1385)^{-}$[11]. Systematic errors were derived from the use of different functional forms to parameterize the signal and the background shape. In addition, guard bands around the $\Lambda$ invariant mass were used to provide an independent estimate of the background mass spectrum.

\section{$7 \quad \Sigma^{ \pm}$Reconstruction}

Experimentally, the charged $\Sigma^{ \pm}$reconstruction is difficult since the only accessible channels are:

$$
\begin{aligned}
\Sigma^{+} & \rightarrow p \pi^{0} \quad(\simeq 51 \%) \\
& \rightarrow n \pi^{+} \quad(\simeq 48 \%) \\
\Sigma^{-} & \rightarrow n \pi^{-} \quad(\simeq 100 \%)
\end{aligned}
$$

The method described here is based on the fact that $\Sigma^{ \pm}$'s have a large decay length $\left(c \tau\left(\Sigma^{-}\right)=4.49 \mathrm{~cm}, c \tau\left(\Sigma^{+}\right)=2.39 \mathrm{~cm}\right)$ and are often measured in the VD. Because they generally decay before the entrance of the TPC, a characteristic signature of a $\Sigma^{ \pm}$track passing through the VD is 3 hits in this detector and no TPC track associated to them. Including the beam position, a measurement of the curvature of the $\Sigma^{ \pm}$trajectory, and thus of its momentum, is obtained. When combined with the measured pion track it gives a signal in the $\Sigma \rightarrow n \pi^{ \pm}$modes. However, there are other sources producing the same topological signature as described above. Their main origin can be categorized (and suppressed) as follows:

- TPC sector boundaries

When a track passes through a dead region of the TPC it is not reconstructed and the 
corresponding hits in the VD can count as a $\Sigma^{ \pm}$candidate. This background was excluded by rejecting any of the above candidates pointing to a dead region within an azimuthal tolerance of $\pm 5^{\circ}$.

- Nuclear interactions

When a track undergoes an inelastic collision with material before the entrance of the TPC, generally more than 2 charged tracks are produced in a momentum range of $0.1<p<2 \mathrm{GeV} / c$. When the collision is after the third VD layer (mainly in the ID trigger layers) the following procedure was used to determine if there has been an interaction:

- a secondary vertex at a radial distance of $11<R<35 \mathrm{~cm}$ is reconstructed with all charged tracks of required momenta, and is kept if its $\chi^{2}$ probability is larger than $1 \%$;

- the $\Sigma^{ \pm}$candidate is extrapolated to radius $R$ and is considered as a nuclear interaction if its impact parameter with respect to the secondary vertex is compatible with the vertex position within three standard deviations.

- Inefficiency on hit association to tracks

When, due to tracking inefficiencies, VD hits are left unassociated, these can mimic a $\Sigma^{ \pm}$candidate. This background is reduced by an extra iteration in which the $\Sigma^{ \pm}$track candidate is rejected if it can be extrapolated and associated to a track element defined by hits in the Inner Detector which are already associated to an existing track.

The track parameters of the surviving candidates (in the following denoted VD-tracks) are defined in the $R \phi$ plane by the coordinates of the associated VD hits. The transverse momentum resolution of these VD-tracks has been estimated from the real data to be $\sigma\left(\frac{1}{P_{t}}\right)=0.05(\mathrm{GeV} / c)^{-1}$. The $R \phi$ component of the $\Sigma^{ \pm}$decay vertex is estimated from the interception of the VD-track with charged tracks reconstructed in the TPC taken as pions. This combinatorial background was reduced by requiring the following for the TPC tracks:

- momentum between 0.1 and $2 \mathrm{GeV} / \mathrm{c}$;

- impact parameter on the $\mathrm{R} \phi$ plane with respect to the primary vertex greater than 5 standard deviations;

- no associated hits in the VD.

The polar direction of the $\Sigma^{ \pm}$candidate was deduced from the coordinates of the primary vertex, which was estimated in this case on an event by event basis, and the charged track coordinates at the $\Sigma^{ \pm}$decay vertex.

A further reduction of the background is obtained by requiring the presence of energy in the Hadron Calorimeter in the direction of the expected neutron. The direction of the neutron, in the two body decay $\Sigma \rightarrow n \pi$, is evaluated from the $\Sigma^{ \pm}$and the $\pi$ momenta. This momentum vector was extrapolated to the Hadronic Calorimeter and a reconstructed hadronic shower was demanded within azimuthal and polar angle tolerances of $\pm 60 \mathrm{mrad}$ and of $\pm 50 \mathrm{mrad}$ respectively.

Figure 5 shows the invariant mass spectra of the $n \pi$ candidates. The right and wrong sign combinations were defined as the same sign and opposite sign for the $\Sigma^{ \pm}$candidates and the daughter pions. A fit to the right sign combination mass spectrum in the region $1.15 \mathrm{GeV} / c^{2}<m<1.3 \mathrm{GeV} / c^{2}$ using a gaussian distribution for the signal and a linear background parameterization resulted in a mean mass of $1187 \pm 2.3 \mathrm{MeV} / \mathrm{c}^{2}$ and a RMS width of $12.9 \pm 2.3 \mathrm{MeV} / c^{2}$ in good agreement with the simulation expectation of $1193 \pm 3$ $\mathrm{MeV} / \mathrm{c}^{2}$ and a width of $12.7 \pm 2.4 \mathrm{MeV} / \mathrm{c}^{2}$ respectively.

Simulation studies have shown that by subtracting the wrong sign combinations from the right sign in the mass range $1.15-1.26 \mathrm{GeV} / \mathrm{c}^{2}$, the true number of generated particles is 
recovered to within $15 \%$, consistent with the error coming from the simulation statistics. After applying the same procedure to the data, an excess of $476 \pm 39$ events in the region of $1.15<M_{n \pi}<1.26 \mathrm{GeV} / c^{2}$ was found in the right sign combination mass spectrum.

The efficiency for $\Sigma^{ \pm}$reconstruction has been studied on a simulated sample of 10000 $\Sigma^{ \pm}$'s produced in $q \bar{q}$ events. It is determined from:

- reconstruction of the $\Sigma^{ \pm}$track in the VD: $10 \%$;

- rejection of the backgrounds (see above): $40 \%$;

- reconstruction and selection of the pion: $70 \%$;

- tagging of the neutron in HCAL: $15 \%$.

Note that the first value includes the geometrical acceptance, the probability for the $\Sigma^{ \pm}$to decay after the last VD layer (different for $\Sigma^{+}$and $\Sigma^{-}$), efficiency of the silicon modules, and the efficiency of the reconstruction algorithm.

The integrated reconstruction efficiency was found to be $0.4 \pm 0.1 \%$

\section{Cross Sections and Correlated Baryon Production.}

The differential cross sections $\left(1 / N_{\text {had }} \times d N / d x_{p}\right)$ for $\Xi^{-}$, and $\Sigma(1385)^{ \pm}$are tabulated in Tables 1 and 2.

In Figure 6 the measured momentum distributions of the above particles is compared with the JETSET 7.3 prediction. In the same figure the published $\Lambda$ differential cross section [5] is included for completeness. Although the $\Lambda$ momentum spectrum indicates a softer behavior than the one predicted by JETSET the $\Xi^{-}$data points agree within errors with the JETSET prediction.

The total $\Xi^{-}$production yield is calculated by integrating the normalized differential cross sections. Assuming that the fraction of $\Xi^{-}$'s in the unobserved momentum range is the same as in JETSET implies an $11 \%$ correction to the above number. This extrapolation contributes with a $5 \%$ systematic error ${ }^{2}$. Nevertheless, the background parameterization error of $5 \%$ and the simulation statistical error of $4.5 \%$ dominate the systematic effects, whilst the uncertainty due to the $\Lambda$ branching ratio to $\mathrm{p} \pi$ adds only $0.5 \%$. The number of $\Xi^{-}$per hadronic $Z$ decay is thus found to be:

$$
<\Xi^{-}>=0.0250 \pm 0.0009 \pm 0.0021
$$

which is consistent with previous LEP measurements [12,4]. JETSET describes this measured yield successfully (0.0265) whilst the HERWIG 5.7 model overestimates the $\Xi^{-}$ production by a factor of more than two (0.058).

The differential momentum cross sections of the other baryons are dominated by the statistical errors. The integrated efficiencies are used in the following to obtain the production yields.

The $\Xi(1530)^{0}$ was measured to be produced at a rate of:

$$
<\Xi(1530)^{0}>=0.00461 \pm 0.0004 \pm 0.0004
$$

per hadronic Z decay, in agreement with Ref. [12] and consistent with the JETSET prediction of 0.0052 whilst a much higher cross section of 0.027 is predicted by HERWIG. The systematics are estimated as a $5 \%$ error due to the extrapolation to the unobserved

\footnotetext{
${ }^{2}$ In the following the several contributions to the systematic errors are quoted as a percentage of the measured value and are combined in quadrature to give the total systematic error.
} 
kinematical region, a $5 \%$ error due to the background parameterization and an error coming from simulation statistics of $7 \%$.

The $\Sigma(1385)^{ \pm}$measured yield of:

$$
<\Sigma(1385)^{ \pm}>=0.0382 \pm 0.0028 \pm 0.0045
$$

lies below the predictions of the JETSET (0.073) and HERWIG (0.134) but is consistent with the measurement in Ref. [12]. The systematics for this measurement consist of a $7.7 \%$ background parameterization error, a $7.1 \%$ error from simulation statistics, a $2.3 \%$ error from branching ratio uncertainties and a 5\% error coming from the kinematical extrapolation.

The $\Sigma^{ \pm}$production rate was established for the first time in the $\mathrm{Z}$ hadronic decays to be:

$$
<\Sigma^{ \pm}>=0.170 \pm 0.014 \pm 0.061
$$

in agreement with the JETSET (0.18) and HERWIG (0.14) values. The systematics are estimated as a $21 \%$ error due to the background subtraction (and signal estimation) technique, a $5 \%$ error coming from the kinematical extrapolation, a $24 \%$ error due to the efficiency estimation from the simulated sample and an additional $16 \%$ error due to the VD and HCAL modelling in the detector simulation. This last systematic error component has been estimated by comparing the number of tracks per event with $3 \mathrm{VD}$ hits in the data and the simulation and by studying the energy deposition in the HCAL from protons of known momenta which have been tagged as $\Lambda$ decay products.

The contributions of the different systematic errors to the above measurements are summarized in Table 3 . Table 4 is a compilation of the measured strange baryon yields and the hadronization model predictions.

Our measurement on the $\mathrm{K}^{0}$ production rate [6] is also included for the sake of the following discussion.

The general picture can be summarized in the following three statements:

- Good agreement between the published LEP results[12,4].

- HERWIG 5.7 overestimates the strange baryon production and especially the decuplet yields.

- JETSET 7.3 describes the octet baryon and the $\Xi(1530)^{0}$ production sufficiently well but fails to reproduce the $\Sigma(1385)^{ \pm}$yield.

In JETSET the popcorn mechanism modifies the production of strange baryons by an additional strangeness suppression and an enhancement of the decuplet production. In parallel the production of a strange meson between two baryons while conserving baryon number locally does not require both the baryons to carry strangeness. It has been shown $[13,5]$ that the correlated production of $\Lambda \bar{\Lambda}$ is softer in JETSET without popcorn than that measured in LEP. The correlated signal of $\Xi^{-}$and $\Lambda$ presented in section 4 is used to measure the probability of baryon and strangeness correlation. Assuming that the efficiency of reconstructing a baryon is independent of the production and identification of the other baryon in the same event, the integrated efficiencies of the $\Xi^{-}$and $\Lambda$ reconstruction were used as described in our previous publication [5] to obtain the following correlated baryon anti-baryon production rates per $\mathrm{Z}$ hadronic decay:

$$
<\Xi^{-} \bar{\Lambda}+\bar{\Xi}^{+} \Lambda>=0.0085 \pm 0.0018 \pm 0.0019
$$

The systematic errors are calculated using the uncertainties in the $\Xi^{-}$and $\Lambda$ production measurements. This average correlated production rate, when normalized to the measured 
total $\Xi^{-}$yield, corresponds to a $34 \% \pm 7 \% \pm 7 \%$ probability of a $\bar{\Lambda}(\Lambda)$ being produced with a $\Xi^{-}\left(\bar{\Xi}^{+}\right)$baryon. The JETSET prediction for correlated $\Xi^{-} \bar{\Lambda}$ or $\Xi^{+} \Lambda$ pair varies as $0.0218,0.0141$ and 0.0093 per $\mathrm{Z}$ hadronic decay ${ }^{3}$ for values of the popcorn parameter of $0,0.5$ and 0.9 respectively. Although tagging events containing a $\Xi^{-}$gives statistical limitations which compromise the viability of rapidity studies, so that the locality of baryon number conservation cannot be proven, the measured correlated production of of the $\Xi^{-}$and $\Lambda$ favours large values of the popcorn parameter in JETSET.

\section{$9 \quad$ Event Topology}

The average charged particle multiplicity has been found [14] higher in multi-jet (three or more jet) topologies than in 2 jet events. From this measurement one might expect similar relative yields to be observed in strange particle production. An additional topology dependent production of strangeness could be expected given that additional strange quarks are produced during the showering of hard gluons.

The definition of the jet multiplicity is biassed by the choice of a) the recombination algorithm and $b$ ) the choice of of the jet resolution parameter. Comparison between measurements and theoretical predictions can be made for a specific algorithm and a range of values of the resolution parameter. The correspondence between hadrons and partons has to be taken as that given by the hadronization model built in the simulation. In Figure 7 the jet multiplicities of the hadronic events used in this analysis are compared with the JETSET 7.3 parton shower prediction (tuned as in [9]) at the hadron level. Both the JADE [15] and LUCLUS [2] recombination schemes were used for jet finding by varying the values of their resolution parameters $y_{c u t}$ and djoin in the range of 0.005 to 0.1 and 1.0 to 10 respectively. Only charged particles were used for the jet definition. The extracted multiplicities have been left uncorrected for detector effects. Instead, the detailed detector simulation was utilized to treat the JETSET predictions identically to the real data (this choice is dictated for the needs of the following analysis).

Each of the subsets of the $Z$ hadronic decays which have been categorized as 2 jet or multi jet events, was processed to identify $\mathrm{K}^{0}, \Lambda$ and $\Xi^{-}$. The $\mathrm{K}^{0}$ were reconstructed through their decay to $\pi^{+} \pi^{-}$with the same criteria as in our previous publication [6], while for the strange baryons, the reconstructed samples of this analysis were used. The same procedure was repeated on simulated hadronic events.

For each of these two event categories, the efficiencies (integrated over the whole momentum region) were estimated from the simulation as a function of the jet resolution parameter. In these efficiencies are included the correction factors coming from the unobserved kinematical regions. The average production rates of the above particles per 2 jet and multi-jet event are shown in Figure 8 as a function of the resolution parameters. The errors correspond to the quadratic sum of the statistical error, the systematic error due to the background estimation and the contribution of the simulation statistics. The other systematic errors due to the kinematical extrapolation and branching ratios are $0.9 \%, 4.5 \%$, and $5 \%$ for the $\mathrm{K}^{0}, \Lambda$, and $\Xi^{-}$respectively but these are not included in the above errors. The JETSET predictions are shown as broken curves in the above figures. The solid curves represent the JETSET values scaled to give the agreement with the data for the total yield per hadronic Z decay (see Table 4).

It has to be emphasized that in the jet finding procedure, the migration of one topological category into another due to detector effects has not been taken into account. However in the trivial case where the migration probabilities are independent of the

\footnotetext{
${ }^{3}$ Corresponding to $62 \%, 53 \%$ and $44 \%$ probabilities for correlated productions respectively.
} 
strange particle production, the corrections to the production yields are only functions of these migration factors. In the opposite case where the existence of a strange particle biases the migration probabilities, e.g. if the strange particle production is enhanced in hard gluonic showers, the correction factors must be evaluated as a function of the kinematical parameters of the jets and the strange particle species under study. To avoid such uncertainties, the JETSET predictions are referred to jet multiplicities found in the same way as in the real data i.e. by employing the detailed detector simulation. The non-scaled JETSET predictions of the $\mathrm{K}^{0}$ and $\Lambda$ production are correspondingly higher than the measurements for both jet topologies. Consequently, the difference in the total production rate between JETSET and the data cannot be assigned preferentially to one or the other of the topological categories.

There is a statistically significant depletion of the $\mathrm{K}^{0}$ measured yield in the 2 jet events relative to the scaled predictions. This discrepancy is evident for very low jet resolution values, for which the influence of details in the simulation of the fragmentation and decay chains could be important, but it is sizeable also at intermediate resolution values (about six standard deviations for djoin $=3 \mathrm{GeV}$ and more than three standard deviations for djoin $=4 \mathrm{GeV}$ ). A similar trend is shown in the $\Lambda$ and $\Xi^{-}$production. The relative production rates of the $\mathrm{K}^{0}, \Lambda$ and $\Xi^{-}$in multi-jet events with respect to the same production in 2 jet events are shown in Figure 9. The comparison of the above ratios between data and JETSET is independent of the scaling of the JETSET prediction. The above figures also include the ratio of the average charged particle multiplicities in multijet relative to 2 jet topologies, extracted from our previous publication [14]. The ratio of the multi-jet to two jet topological yields, for these hadrons, agrees within errors with the ratio of the average charged particle multiplicity for these topologies. However, the above discrepancies with the JETSET predictions are still seen, especially for the $\mathrm{K}^{0}$ sample ${ }^{4}$. This analysis finds that the production of strangeness depends on the event topology in a manner that is not quantitatively described by JETSET.

\section{Conclusions}

The production at the $\mathrm{Z}$ resonance of the $\Xi^{-}, \Xi(1530)^{0}, \Sigma(1385)^{ \pm}$and $\Sigma^{ \pm}$, has been measured and the strangeness production dependence on the event topology has been studied.

The differential momentum cross section of the $\Xi^{-}$has been found to agree within errors with the prediction of the JETSET 7.3 hadronization model. More statistics are required to draw any conclusions concerning the measured momentum distributions of the other baryons studied in this analysis.

The total average baryon production rate per $\mathrm{Z}$ hadronic decay are found to be:

$$
\begin{aligned}
\left\langle\Xi^{-}\right\rangle & =0.0250 \pm 0.0009 \pm 0.0021 \\
\left\langle\Xi(1530)^{0}>\right. & =0.0041 \pm 0.0004 \pm 0.0004 \\
\left\langle\Sigma(1385)^{ \pm}\right\rangle & =0.0382 \pm 0.0028 \pm 0.0045 \\
\left\langle\Sigma^{ \pm}\right\rangle & =0.170 \pm 0.014 \pm 0.061
\end{aligned}
$$

A signal of simultaneous baryon number and strangeness compensation is found by measuring the correlated production of $\Xi^{-}$and $\Lambda$ to be:

\footnotetext{
${ }^{4}$ In Figure 9a the $y_{c u t}=0.005$ point lies outside the figure at a value of $3.315 \pm 0.120$.
} 


$$
<\Xi^{-} \bar{\Lambda}+\bar{\Xi}^{+} \Lambda>=0.0085 \pm 0.0018 \pm 0.0019
$$

This correlated production rate favors large values of the popcorn probability.

The relative production rate of the $\mathrm{K}^{0}, \Lambda$ and $\Xi^{-}$in multi-jet events compared with their production in 2 jet events gives the same ratio within errors as that for the averaged charged particle multiplicities for these topologies. The measured $\mathrm{K}^{0}$ topological yields diverge from the JETSET predictions at small resolution parameter values. However, the corresponding results for $\Lambda$ and $\Xi^{-}$production, although suggesting a similar trend, are consistent with the JETSET predictions within errors.

\section{Acknowledgements}

We are greatly indebted to our technical collaborators and to the funding agencies for their support in building and operating the DELPHI detector, and to the members of the CERN-SL Division for the excellent performance of the LEP collider.

\section{References}

[1] For a recent review on diquarks, see for example M. Anselmino et al., Rev. Mod. Phys. 65 (1993) 1199.

[2] T. Sjöstrand, Comp. Phys. Comm. 27 (1982) 243, ibid. 28 (1983) 229.

[3] G. Marchesini and B.R. Webber, Nucl. Phys. B238 (1984) 1.

[4] P. Abreu et al. (DELPHI Collaboration), Phys. Lett. B275 (1992) 231.

[5] P. Abreu et al. (DELPHI Collaboration), Phys. Lett. B318 (1993) 249.

[6] P. Abreu et al. (DELPHI Collaboration), "Production Characteristics of $\mathrm{K}^{0}$ and Light Meson Resonances in Hadronic Decays of the Z", CERN-PPE/94-130 (August 1994), to be published in Z. Phys. C.

[7] P. Aarnio et al. (DELPHI Collaboration), Nucl. Instr. and Meth. A303 (1991) 233.

[8] DELSIM User's Guide, DELPHI 89-15 PROG 130, CERN, February 1989.

DELSIM Reference Manual, DELPHI 89-68 PROG 143, CERN, September 1989.

[9] W. de Boer and H. Fürstenau, IEKP-KA/91-07, Karlsruhe, 1991.

[10] R. Fruhwirth, Nucl. Instr. and Meth. A262 (1987) 444.

[11] Particle Data Group, Review of Particle Properties, Phys.Rev. D50, Part I (1994).

[12] P.D. Acton et al. (OPAL Collaboration), Phys. Lett. B291 (1992) 503.

[13] P.D. Acton et al. (OPAL Collaboration), Phys. Lett. B308 (1993) 415.

[14] P. Abreu et al. (DELPHI Collaboration), Z. Phys. C56 (1992) 63.

[15] W. Bartel et al. (JADE collaboration), Z. Phys. C33 (1986) 23. 
Table 1: $\Xi^{-}$differential cross section.

\begin{tabular}{|c|c|c|c|}
\hline$x_{p}$ Interval & Efficiency & Number of events & $\frac{1}{N_{\text {had }}} \frac{d n}{d x_{p}}$ \\
\hline \hline $0.015-0.050$ & $0.0432 \pm 0.0045$ & $323 \pm 23 \pm 33$ & $0.214 \pm 0.015 \pm 0.031$ \\
$0.050-0.100$ & $0.1158 \pm 0.0085$ & $677 \pm 33 \pm 43$ & $0.122 \pm 0.006 \pm 0.012$ \\
$0.100-0.150$ & $0.0822 \pm 0.0075$ & $256 \pm 21 \pm 16$ & $0.065 \pm 0.005 \pm 0.007$ \\
$0.150-0.200$ & $0.0448 \pm 0.0070$ & $81 \pm 10 \pm 6$ & $0.038 \pm 0.005 \pm 0.006$ \\
$0.200-0.300$ & $0.0127 \pm 0.0040$ & $28 \pm 7 \pm 6$ & $0.022 \pm 0.006 \pm 0.008$ \\
$0.300-0.400$ & $0.0056 \pm 0.0023$ & $7 \pm 3 \pm 2$ & $0.012 \pm 0.005 \pm 0.006$ \\
\hline
\end{tabular}

Table 2: $\Sigma(1385)^{ \pm}$differential cross sections.

\begin{tabular}{|c|c|c|c|}
\hline$x_{p}$ Interval & Efficiency & Number of events & $\frac{1}{N_{\text {had }}} \frac{d n}{d x_{p}}$ \\
\hline \hline $0.05-0.10$ & $0.118 \pm 0.012$ & $1256 \pm 96 \pm 164$ & $0.224 \pm 0.017 \pm 0.037$ \\
$0.10-0.20$ & $0.101 \pm 0.013$ & $855 \pm 49 \pm 63$ & $0.090 \pm 0.005 \pm 0.013$ \\
$0.20-0.30$ & $0.053 \pm 0.007$ & $196 \pm 29 \pm 46$ & $0.038 \pm 0.005 \pm 0.010$ \\
\hline
\end{tabular}

Table 3: Individual error contribution to the average multiplicities.

\begin{tabular}{|c|c|c|c|c|}
\hline Error & $\Xi^{-}$ & $\Xi^{0}(1530)$ & $\Sigma(1385)^{ \pm}$ & $\Sigma^{ \pm}$ \\
\hline \hline Statistical & $3.6 \%$ & $10.0 \%$ & $7.3 \%$ & $8.2 \%$ \\
Background estim. & $5.0 \%$ & $5.0 \%$ & $7.7 \%$ & $21.0 \%$ \\
MC correction & $4.5 \%$ & $7.0 \%$ & $7.1 \%$ & $28.8 \%$ \\
Branching ratio & $0.5 \%$ & $0.5 \%$ & $2.3 \%$ & $<0.3 \%$ \\
MC extrapolation & $5.0 \%$ & $5.0 \%$ & $5.0 \%$ & $5.0 \%$ \\
Total systematic & $8.4 \%$ & $10.0 \%$ & $11.8 \%$ & $36.0 \%$ \\
\hline
\end{tabular}

Table 4: Total production yields in comparison with the phenomenological models predictions.

\begin{tabular}{|c|c|c|c|}
\hline Hadron Type & Number / event & JETSET & HERWIG \\
\hline \hline $\mathrm{K}^{0}$ & $1.962 \pm 0.022 \pm 0.056$ & 2.15 & 2.37 \\
$\Lambda$ & $0.357 \pm 0.003 \pm 0.017$ & 0.373 & 0.421 \\
$\Xi^{-}$ & $0.0250 \pm 0.0009 \pm 0.0021$ & 0.0265 & 0.058 \\
$\Xi(1530)^{0}$ & $0.0041 \pm 0.0004 \pm 0.0004$ & 0.0052 & 0.027 \\
$\Sigma(1385)^{ \pm}$ & $0.0382 \pm 0.0028 \pm 0.0045$ & 0.0730 & 0.134 \\
$\Sigma^{ \pm}$ & $0.170 \pm 0.014 \pm 0.061$ & 0.180 & 0.142 \\
\hline
\end{tabular}




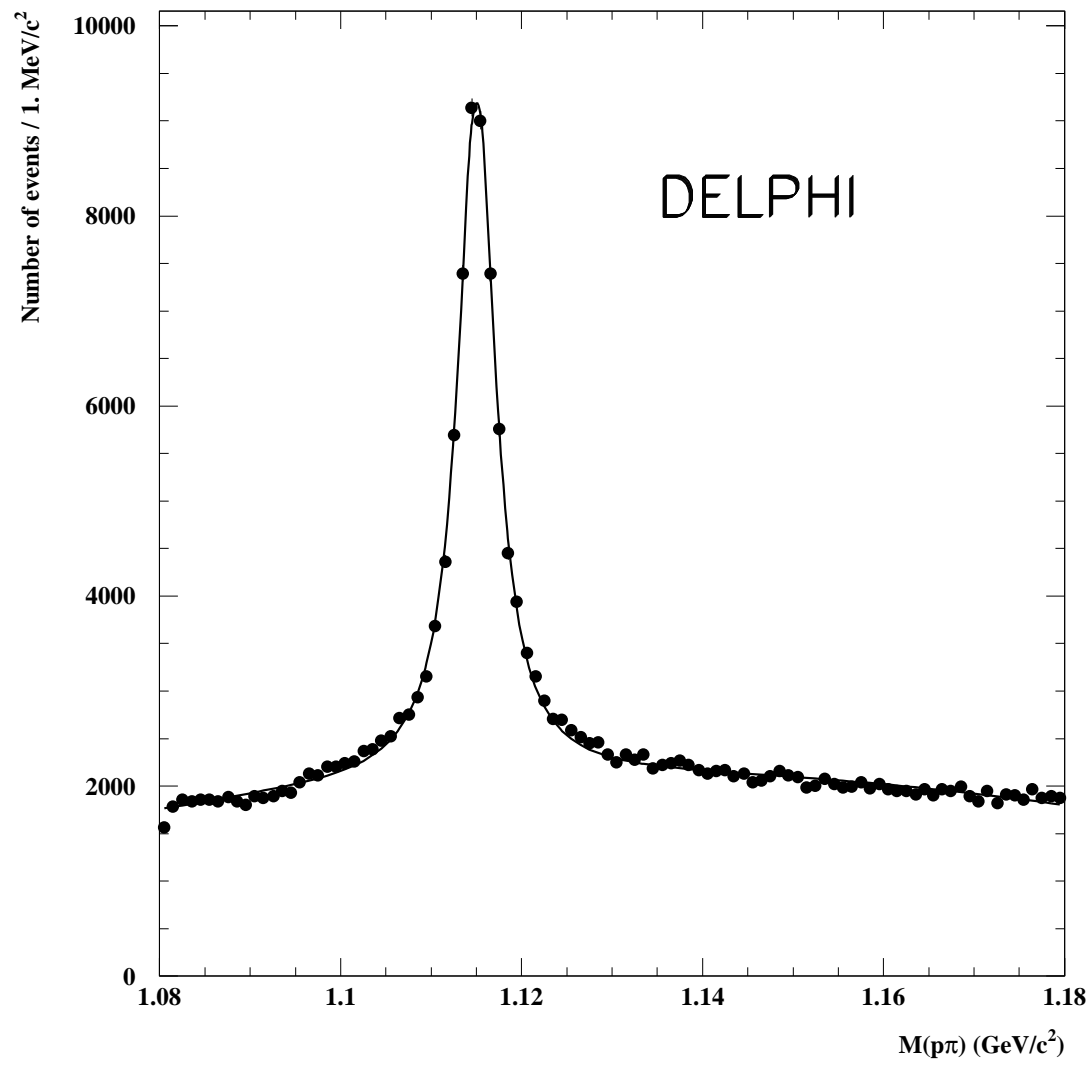

Figure 1: $p \pi^{-}$invariant mass distribution. 

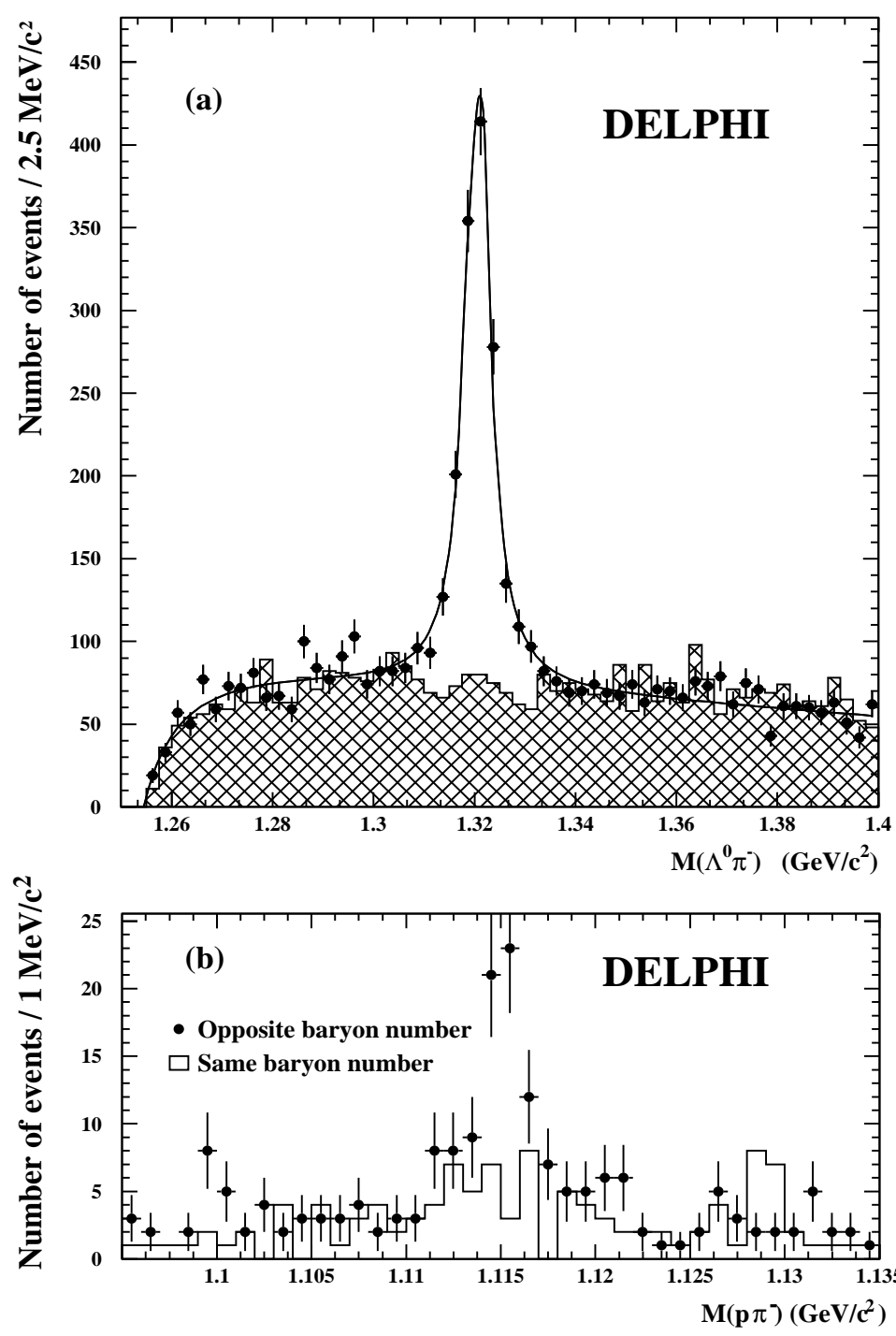

Figure 2: a) The invariant mass distribution of $\Lambda \pi^{-}$and $\bar{\Lambda} \pi^{+}$selected combinations. The shaded histogram represents the mass spectrum of the wrong sign $\left(\Lambda \pi^{+}\right.$and $\left.\bar{\Lambda} \pi^{-}\right)$ combinations. b) The $\bar{p} \pi^{+}\left(p \pi^{-}\right)$invariant mass distribution is shown as data points on top of a histogram representing the $p \pi^{-}\left(\bar{p} \pi^{+}\right)$spectrum for events containing a $\Xi^{-}\left(\bar{\Xi}^{+}\right)$ candidate. 


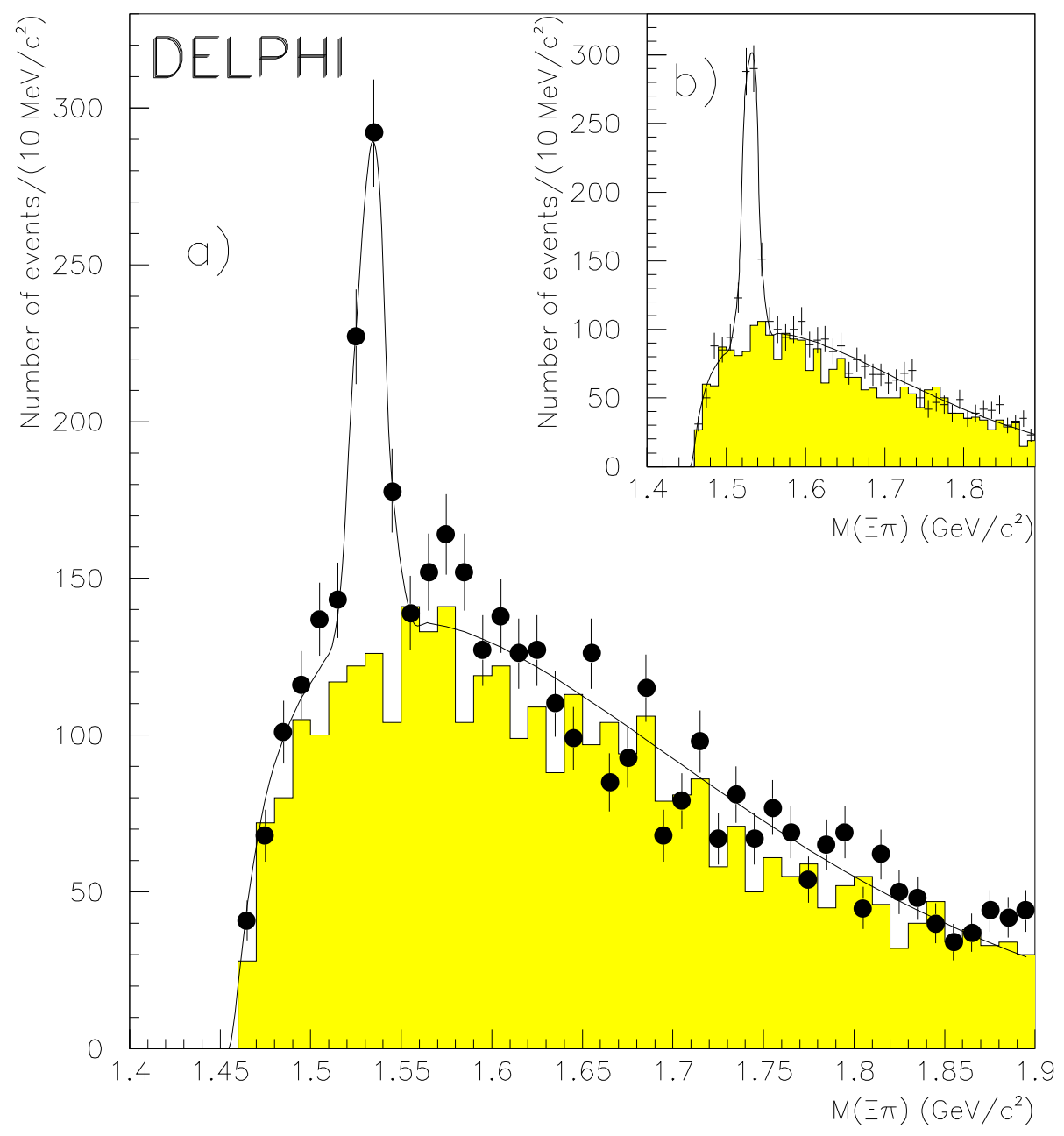

Figure 3: a) $\Xi^{-} \pi^{+}\left(\overline{\Xi^{+}} \pi^{-}\right)$invariant mass distribution. The shaded histogram represents the invariant mass spectrum of the wrong sign combinations. b) The same mass distribution as in a) produced with simulated events. 


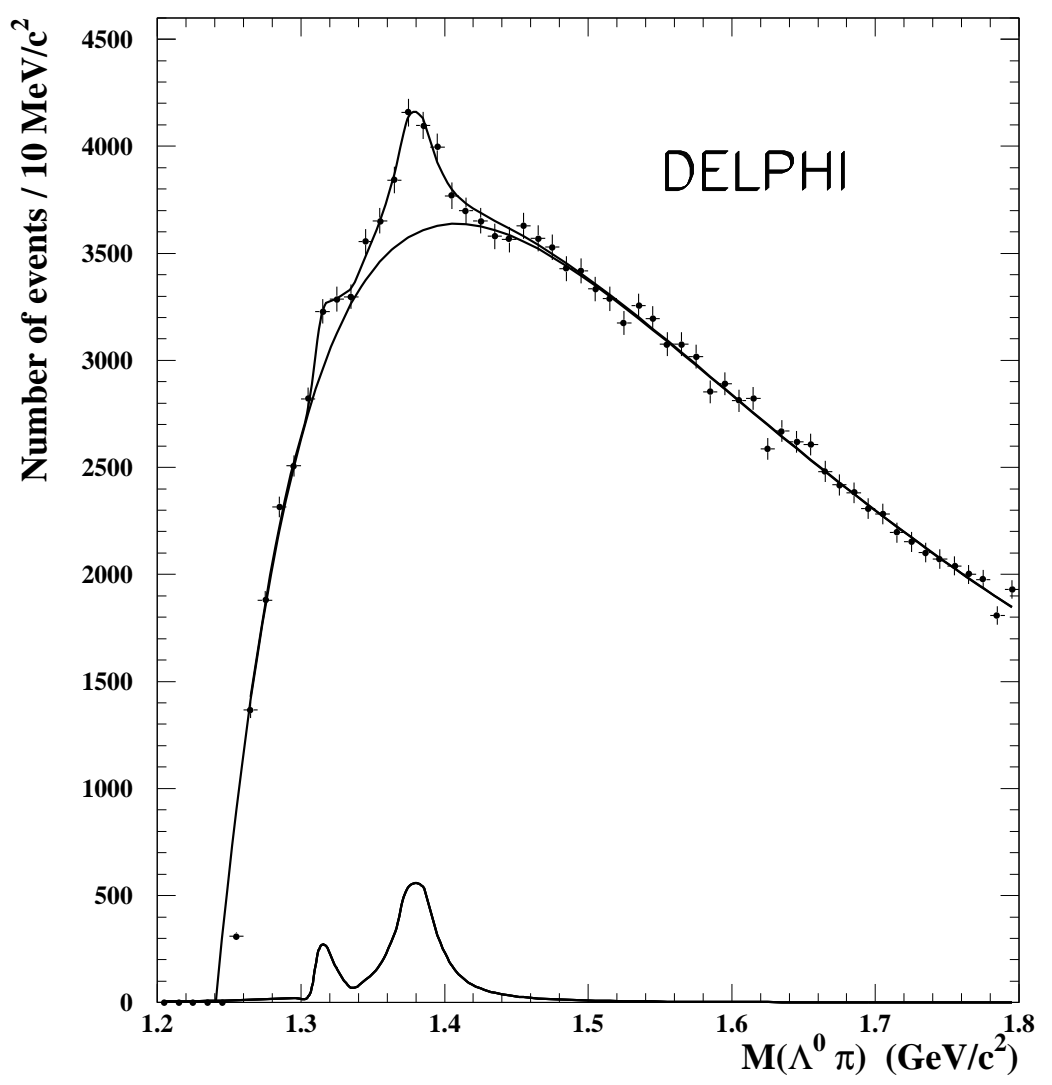

Figure 4: $\Lambda \pi^{+}$(plus $\bar{\Lambda} \pi^{-}$) invariant mass distribution for combinations selected with the criteria of Section 6. Also shown is the mass spectrum of the $\Sigma(1385)^{ \pm}$and $\Xi^{-}$baryons derived from the fit. 


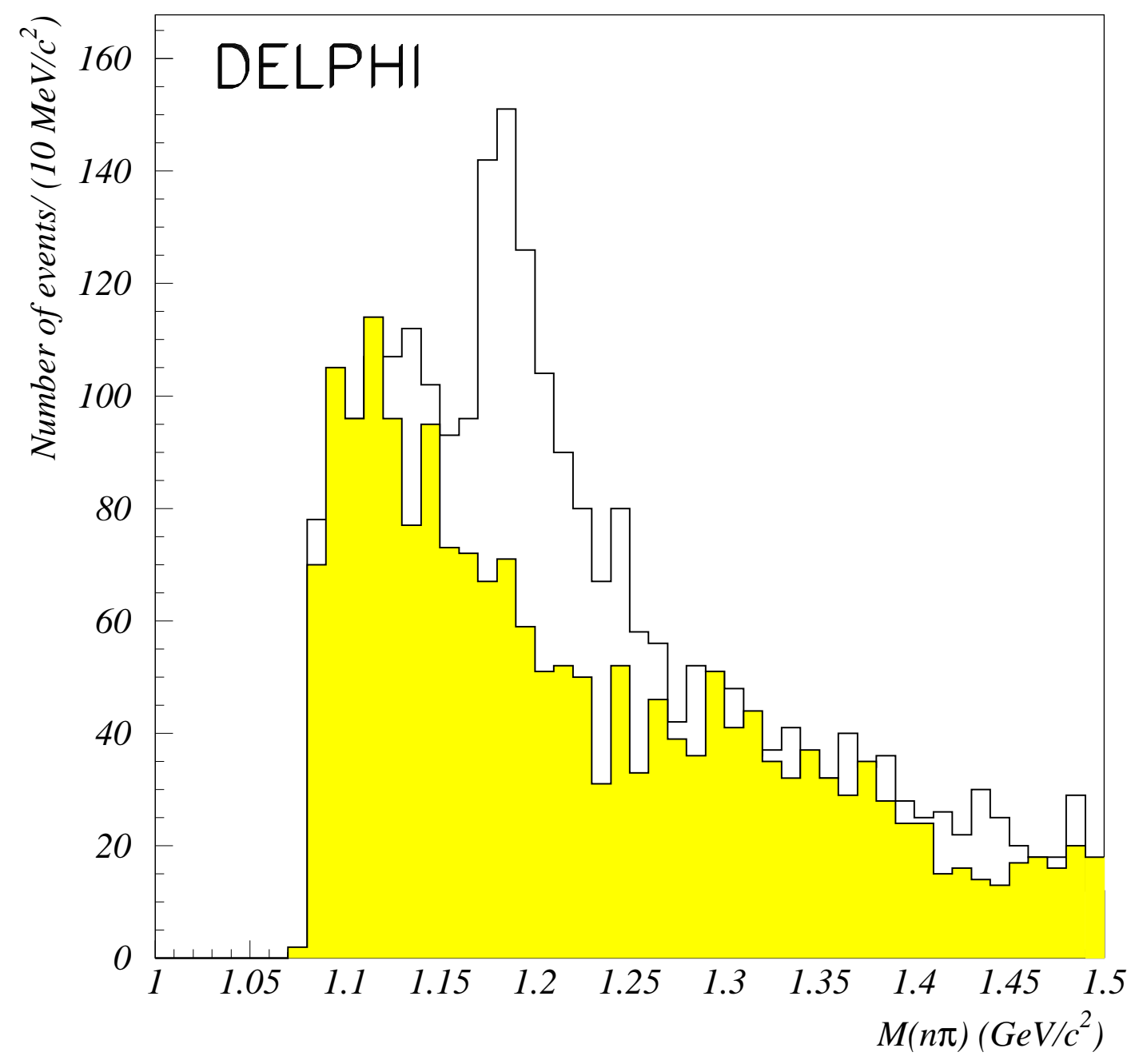

Figure 5: $n \pi$ invariant mass distribution for pair candidates selected as described in Section 7. The grey area shows the wrong-sign combinations. 


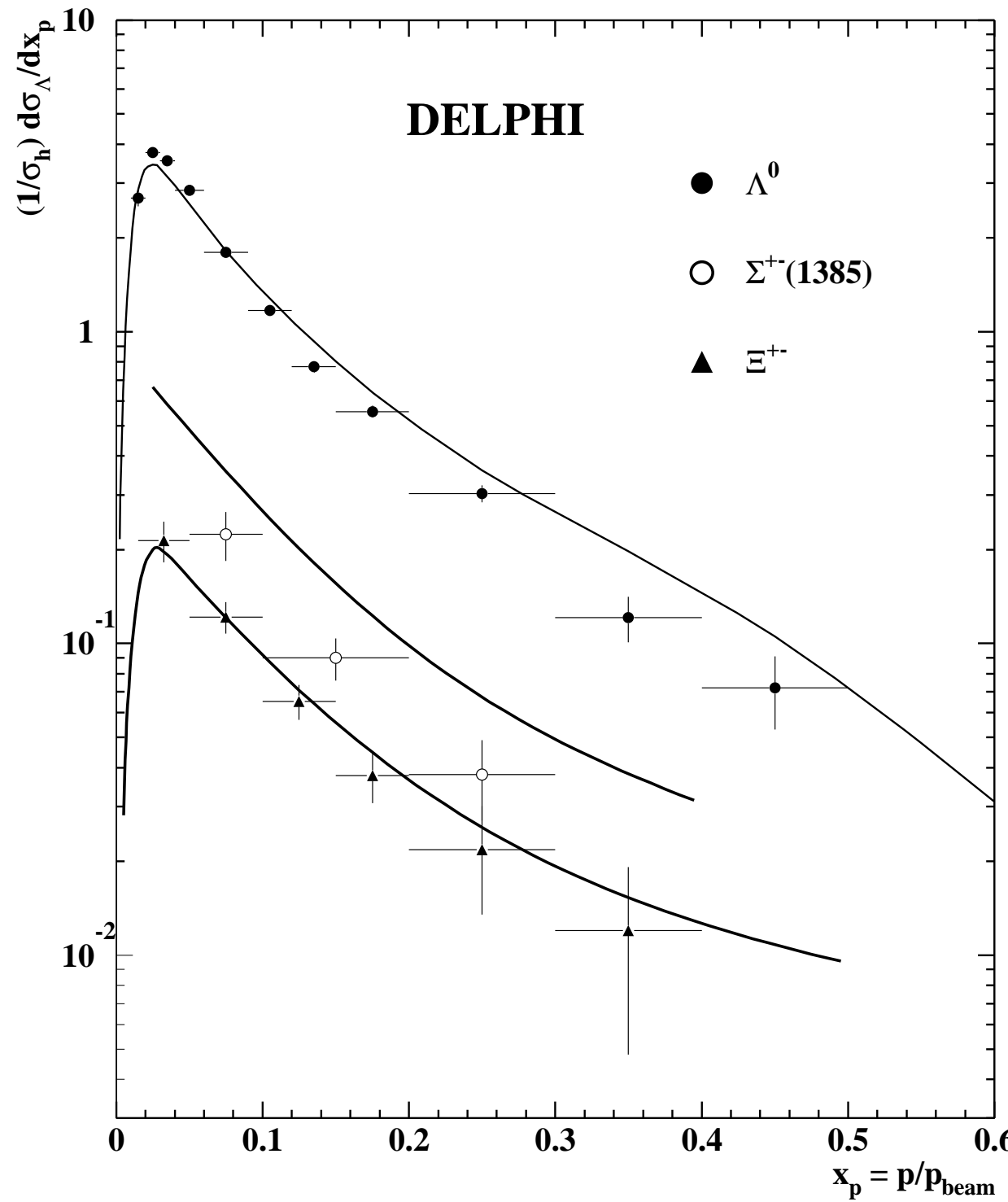

Figure 6: The $x_{p}$ differential distribution for $\Lambda, \Xi^{-}$, and $\Sigma(1385)^{ \pm}$production in comparison with the JETSET prediction. 

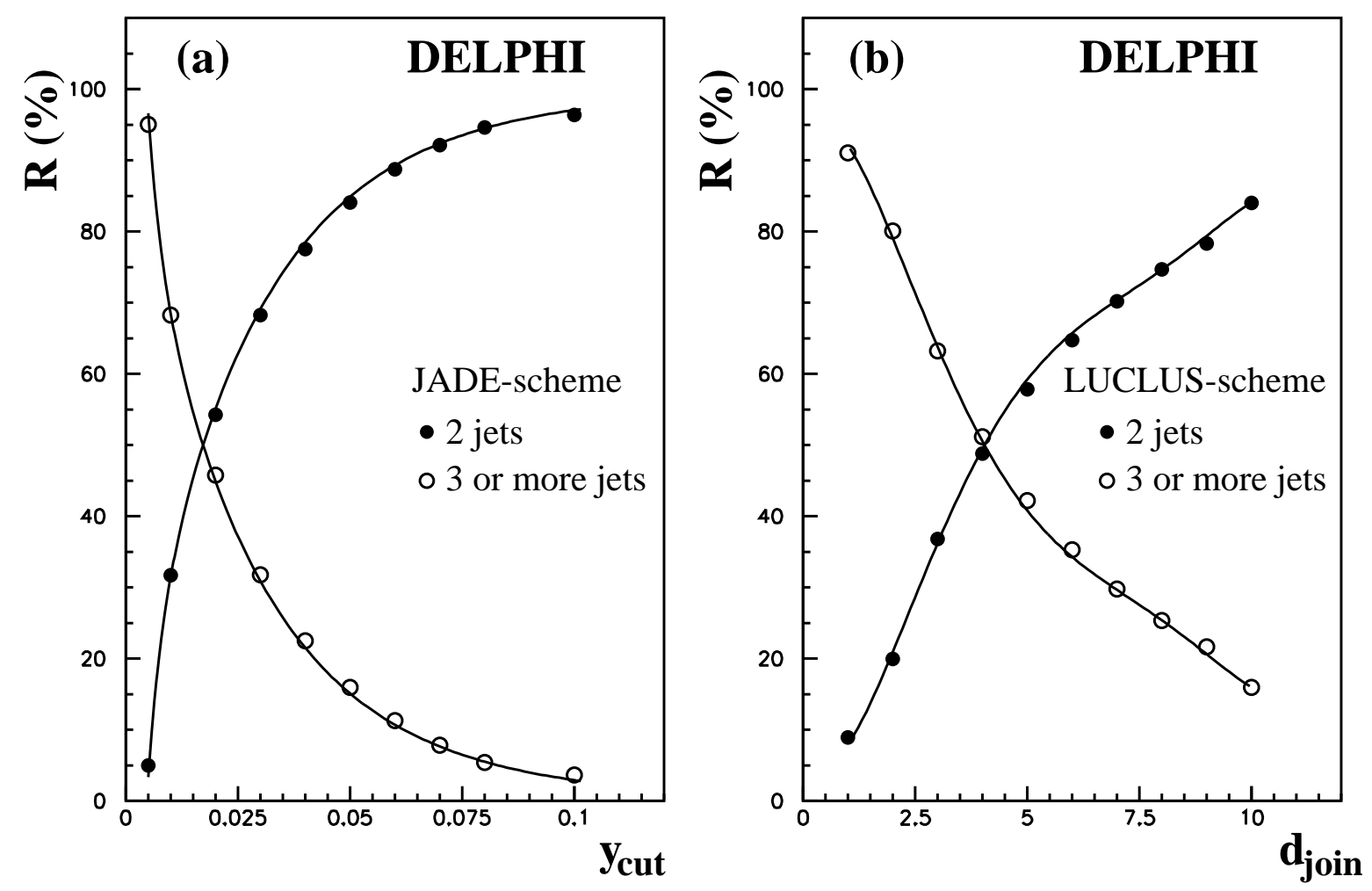

Figure 7: The jet multiplicities found by JADE and LUCLUS recombination schemes as a function of the jet resolution parameter. The solid lines represent the JETSET prediction. 

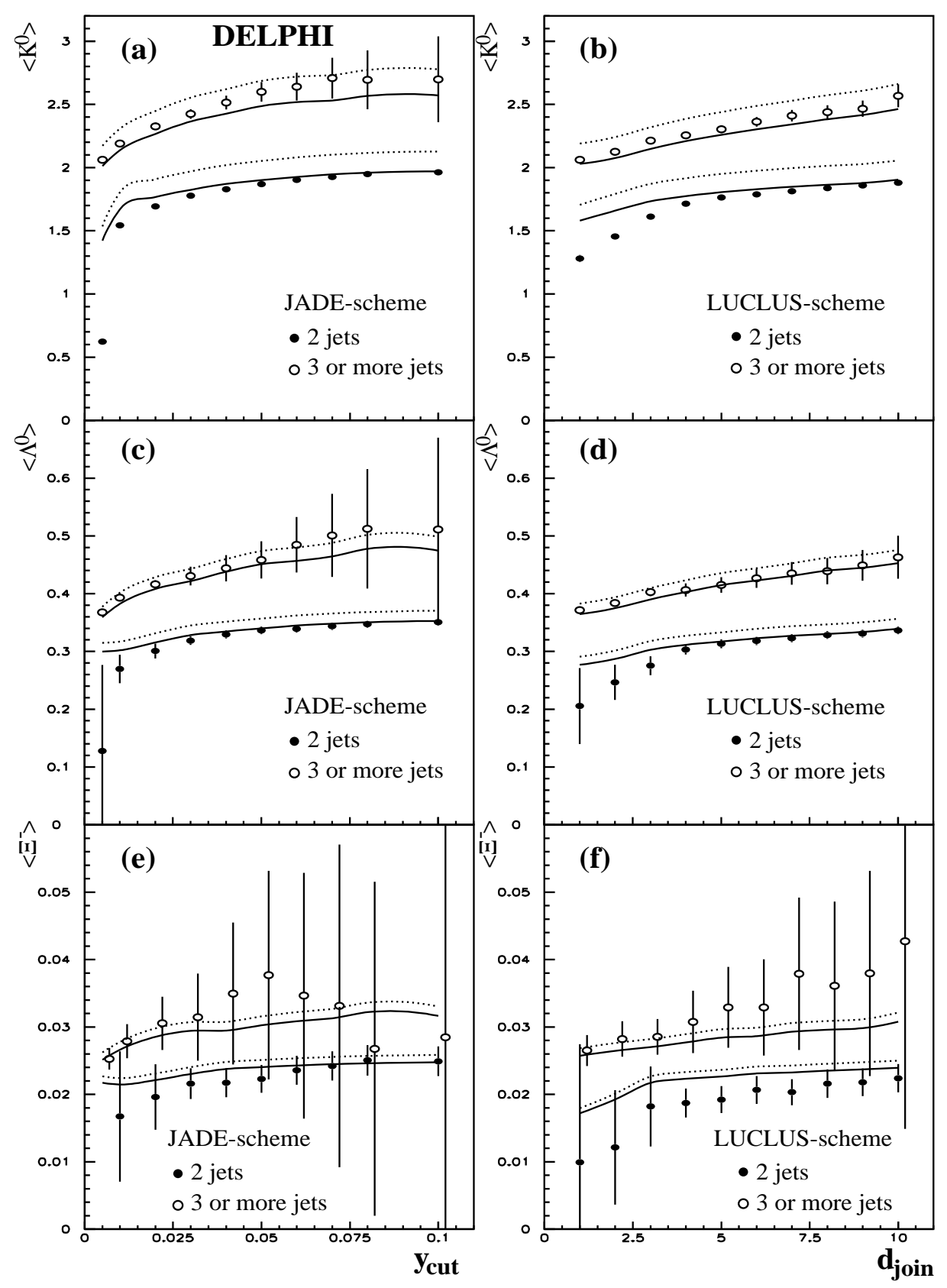

Figure 8: The topological yields as a function of the jet resolution parameter in comparison with the JETSET predictions (broken lines). The solid lines represent the JETSET predictions scaled to match the measured total production rates. (a) and (b) $K^{0}$; (c) and (d) $\Lambda ;(e)$ and (f) $\Xi^{-}$. 

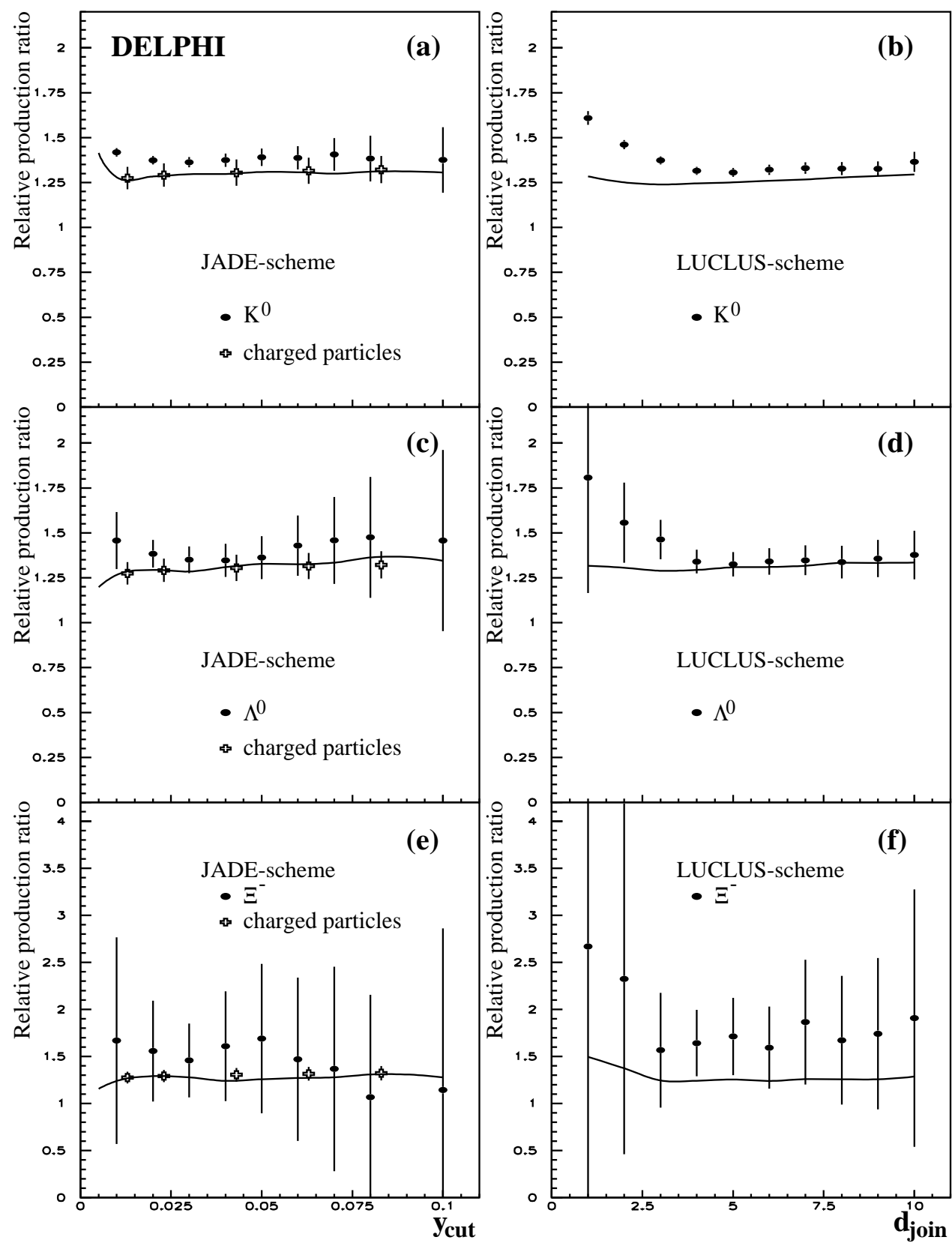

Figure 9: The relative production rates in multi jet events with respect to the production rates in 2 jet events. (a) and (b) $K^{0}$; (c) and (d) $\Lambda ;(e)$ and (f) $\Xi^{-}$. The solid lines represent the JETSET predictions. 